\title{
Current Research in
}

\section{Bioorganic \& Organic Chemistry}

\section{Forensic Analysis of a Confiscated Illicit Heroin Sample}

\author{
Farid A. Badria*, Mona El-Neketi, Hassan-Elrady A. Saad \\ Department of Pharmacognosy, Mansoura University, Mansoura, Egypt
}

"Corresponding author: Farid A. Badria, Department of Pharmacognosy, Mansoura University, Mansoura 35516, Egypt. Tel: +201001762927; Email: faridbadria@gmail.com

Citation: Badria FA, El-Neketi M, Saad HA (2018) Forensic Analysis of a Confiscated Illicit Heroin Sample. Curr Res Bioorg Org Chem: CRBOC-105. DOI: 10.29011/CRBOC -105. 100005

Received Date: 17 February, 2018; Accepted Date: 02 March, 2018; Published Date: 09 March, 2018

\begin{abstract}
Different forensic analytical methods involving physical and microscopical examination, color tests, Thin Layer Chromatography (TLC) and Gas Chromatography/ Mass Spectrometry (GC/MS) were applied for the identification and characterization of an illicit heroin sample confiscated in Egypt. These methods confirmed that the heroin sample under investigation is a poorly manufactured sample, prepared by acetylation with acetic anhydride, badly stored, adulterated and it is of the South West Asian type and suggested to be abused by inhalation through smoking.
\end{abstract}

Keywords: Chromatography-Mass Spectrometry (GC-MS); Confiscated illicit; Heroin; opium alkaloids; TLC

\section{Introduction}

Heroin (Diacetylmorphine) is a semi-synthetic analogue of morphine, prepared by acetylating with acetyl chloride or acetic anhydride. It belongs to the internationally controlled narcotic analgesics with morphine, codeine and other synthetic drugs [1,2]. It was some seventy years after the first isolation of morphine from opium before the synthesis of diacetylmorphine was first reported in 1874. Commercial production by the Bayer Company, who named this new drug heroin, began in 1898. By the beginning of the twentieth century, heroin was widely accepted by the medical profession, and was typically used as a substitute for codeine and morphine in tuberculosis and other respiratory diseases. It was also about this time that heroin first appeared in China. A few years after the 1925 International Convention on Narcotics, international controls began to limit the supply of heroin, and the clandestine manufacture of heroin began [1-3]. No one could have suspected this would become one of the most notorious drugs of our time. The global illicit production of opium (from which heroin is processed) becomes increasingly concentrated in Afghanistan which has been producing three-quarters of the world's illicit opium [4].

Analysis of illicit heroin samples in criminal cases to identify their main active principles, diluents, adulterants and impurities that may adversely affect the abuser's heath is important for judicial purposes, identification of the source of a sample, tracing of distribution routes and identifying new production processes and ascertains whether two or more exhibits came from an identical source [3,5]. Different forensic analytical methods are concerned with heroin analysis these methods include: presumptive color tests3, [6-10]; Thin Layer Chromatography [3,7,11-19]. Without question the gas chromatography interfaced Mass Spectrometer (GC/MS) is one of the most useful tools available to the forensic drug chemist. It is able to provide highly specific spectral data on individual compounds in a complex mixture of compounds, without prior separation of these components [3]. Many GC/MS methods were published for illicit heroin preparations analysis [20-27]. In this report, the analyses of heroin, its impurities and adulterants using color and precipitation tests, TLC, GC/MS are described with the aim of providing information about the origin, the trafficking and synthetic routes of illicit heroin samples.

\section{Experimental}

\section{Material and Reagent}

Drugs

Confiscated heroin sample: It was obtained from seizure number 978 / 1988, Suez- Egypt.

\section{Reference Materials}

Opiate alkaloids 
- Morphine sulfate ampoules $(20 \mathrm{mg} / \mathrm{ml})$ from Misr Co. for Pharmaceutical industry, Egypt.

- Codeine phosphate powder from Supreme organization of Drugs and Medical Requirements, Cairo (Czechoslovakia).

- Papaverine hydrochloride powder from BDH chemical, Ltd. England

- Noscapine: It was isolated from Noscapine Syrup (Agropharm, Buckingham House, church Road, Pann, Bucks. HP10 8LN).

- Heroin (diacetylmorphine) and $\mathrm{O}_{3}$-Monoacetylmorphine were prepared in our laboratory from morphine.

- O6-monoacetylmorphine hydrochloride: United Nations UNIES Vienna international center. Chief, scientific section, DOA/ UNDDP, Howard Street.

- Acetylcodeine: It was prepared in our laboratory from codeine.

The identity of the prepared compounds was confirmed by comparison of their spectral data (UV, IR and EI-MS) with the previously published ones $[2,3,7,10,26,28-30]$

\section{Apparatus}

- Leitz Wetzlar microscope GMBH fitted with camera Lucida (Germany).

- Precoated silica gel G60F254 (20 x $20 \mathrm{~cm} \times 0.2 \mathrm{~mm}$ thick) on aluminum (E-Merck and Machery-Nagel, Germany), Ultraviolet lamp operating at $\lambda 254,366 \mathrm{~nm}$ (Desaga, Germany) for location of TLC spots, Glass jars of different sizes, micropipettes for spotting.

- GC/MS was Carried out at National Research Center, Dokki, Cairo, Egypt on GC/MS Finnigan mat SSQ7000 chromatograph with Digital DEC 3000 Work station. Helium was used as carrier gas at a flow rate of $1.6 \mathrm{ml} / \mathrm{min}$ and column head pressure was 13 Psi. The gas chromatograph was coupled with mass detector (MS) at $70 \mathrm{eV}$ in EI ionization mode.

\section{Chemicals and Reagents}

The following reagents were used during the course of this work:

- Derivatizing reagents: N-methyl-Trimethyl Silyltrifluoro acetamide (MSTFA) reagent for silylation was purchased from Sigma USA.
- All reagents and solvents for TLC separation were of analytical grade and purchased from (Adwik, Egypt), while those for GC/MS analysis were of spectroscopic grades.

- Color reagents: Marquis, Froehde, Meck,s reagent, Chromatographic Spray reagents: Dragendroff,s and acidified Potassium iodoplatinnate were prepared according to United Nations publications [3].

\section{General Procedure}

\section{Microscopic Characters, Color, Solubility and Precipitation} (Anion) Tests

\section{Sample Preparation}

- Microscopic characters: About $0.5 \mathrm{~g}$ of the heroin sample was dissolved by gentle shaking in $10 \mathrm{ml}$ of distilled water. The obtained solution was centrifuged, the clear supernatant was separated, transferred to a clean test tube and reserved for carrying out the color tests (solution 1), and the residue was subjected to exhaustive washing with water to be suitable for microscopic examination [3]. Several mounts of vegetable debris were prepared in water, chloral hydrate, and phloroglucinol and conc. $\mathrm{HCl}$ and examined under the microscope.

- For color tests: The previously reserved clear supernatant of the heroin sample was used (solution 1). Other portions of heroin were dissolved in methanol [ $1 \mathrm{mg}$ in $0.5 \mathrm{ml}]$ for Oliver test 8 and $[10 \mathrm{mg}$ in $1 \mathrm{ml}$ ] for Murexide test 31 then centrifuged. With a disposable pipette, the supernatant was drawn into a clean test tube (solution $2 \& 3$ ).

- For solubility test: About $100 \mathrm{mg}$ of illicit heroin sample was dissolved in $0.5 \mathrm{ml}$ distilled water (to test its solubility in water). The same steps were repeated but water was replaced by ethanol (to test the presence of carbohydrates) $[2,7]$.

- For precipitation (Anion) test: About $1 \mathrm{~g}$ of the illicit heroin sample was dissolved in approximately $5 \mathrm{ml}$ distilled water and centrifuged. The supernatant was removed to a clean test tube (solution 4) for precipitation test $[2,3,7]$.

\section{Color and Precipitation (Anion) Tests Procedures}

The procedures for presumptive color and precipitation (Anion) tests are cited in (Table 1). 
Citation: Badria FA, El-Neketi M, Saad HA (2018) Forensic Analysis of a Confiscated Illicit Heroin Sample. Curr Res Bioorg Org Chem: CRBOC-105. DOI: 10.29011/ CRBOC -105. 100005

\begin{tabular}{|c|c|c|c|}
\hline Test Name & Method & Use & Ref \\
\hline \multicolumn{4}{|c|}{ Presumptive color tests: } \\
\hline $\begin{array}{l}\text { Marquis, Froehde, } \\
\text { Meck's and nitric acid } \\
\text { test }\end{array}$ & $\begin{array}{l}\text { One drop of heroin solution (solution 1) was placed in each well in a spot tile } \\
\text { followed by adding } 3 \text { drops of each Marquis, Froehde, Meck's and nitric acid } \\
\text { reagent. }\end{array}$ & $\begin{array}{l}\text { Heroin and opium } \\
\text { alkaloid detection }\end{array}$ & {$[2,3,6-8]$} \\
\hline Oliver test: & $\begin{array}{l}\text { A trace of copper sulfate solution ( } 1 \% \text { in water) was added by means of a glass } \\
\text { rod wetted with copper sulfate to solution } 2 \text {. The solution was stirred, and } 1 \mathrm{ml} \text { of } \\
3 \% \text { hydrogen peroxide and } 1 \mathrm{ml} \text { of conc. ammonium hydroxide were added and } \\
\text { shaken. }\end{array}$ & Heroin detection & {$[6,8]$} \\
\hline Murexide test & $\begin{array}{l}\text { One } \mathrm{ml} \text { of } 10 \% \mathrm{HCl} \text { was mixed with } 1.0 \mathrm{ml} \text { of solution } 3 \text { to which } 0.1 \mathrm{~g} \text {. } \\
\text { Potassium chlorate was added in white porcelain dish and evaporated to dryness } \\
\text { on a water bath. The residue was exposed separately to amm. vapor. }\end{array}$ & Caffeine detection & {$[31]$} \\
\hline \multicolumn{4}{|c|}{ Test for anions } \\
\hline \multirow{4}{*}{ Silver nitrate test } & \multicolumn{2}{|c|}{ A portion of solution 4 was added to few drops of silver nitrate $5.0 \% \mathrm{w} / \mathrm{v}$ solution. } & \multirow{5}{*}[2]{} \\
\hline & $\begin{array}{l}\text { Chloride precipitate is insoluble in conc. Nitric, soluble in dilute ammonia } \\
\text { solution, from which it can be precipitated by addition of nitric acid. }\end{array}$ & Chloride detection & \\
\hline & Tartarate precipitate is soluble in nitric acid. & Tartarate detection & \\
\hline & Citrate precipitate is soluble in nitric acid. & Citrate detection & \\
\hline Acetic anhydride test & $\begin{array}{l}\text { A small amount of illicit heroin sample was heated with } 0.5 \mathrm{ml} \text { acetic anhydride } \\
\text { at } 80^{\circ} \mathrm{C} \text { for } 10 \text { minutes. }\end{array}$ & Citrate detection & \\
\hline Barium chloride test & $\begin{array}{l}\text { Another portion of solution } 4 \text { was added to few drops of barium chloride } 10 \% \\
\text { w/v solution. Sulfate precipitate is insoluble in } \mathrm{HCl} \text {. }\end{array}$ & $\begin{array}{l}\text { Sulfate anion } \\
\text { detection }\end{array}$ & {$[2,3]$} \\
\hline
\end{tabular}

Table 1a: Procedures for presumptive color and precipitation tests of the heroin sample.

\section{Thin Layer Chromatography (TLC)}

\section{Developing solvents}

System A: Chloroform-n-hexane-triethylamine (9:9:4 v/v)16

System B: Chloroform-Methanol (9:1 v/v)7

\section{Sample and standard solutions preparation}

Five mgs heroin sample were dissolved in $1 \mathrm{ml}$ methanol and centrifuged. The supernatant was separated into a clean vial, from which $3 \mu 1$ were spotted to the TLC plate. Morphine sulfate, codeine phosphate, papaverine hydrochloride, O6-monoacetylmorphine hydrochloride, acetyl codeine, heroin and caffeine were made at conc. $5 \mathrm{mg} / \mathrm{ml}$ methanol. Noscapine was made at the same concentration but in chloroform [3].

\section{TLC separation}

The methanolic solution of heroin sample and standard solutions were examined by TLC on pre-coated silica gel plates using solvent systems A and B. The developed plates were air dried and visualized under UV at $254 \mathrm{~nm}$ followed by spraying separately with Dragendroff,s and acidified potassium iodoplatinnate reagents.

\section{Gas Chromatography Interfaced Mass Spectrometer (GC/ MS)}

\section{Sample Preparation}

Illicit heroin sample was subjected to several sample preparations either as total or neutral fraction for full sample characterization

\section{A) Total illicit heroin sample}

1) Direct analysis: Five mgs of illicit heroin sample were dissolved in $1 \mathrm{ml}$ methanol- chloroform $(4: 1 \mathrm{v} / \mathrm{v})$, sonicated for $10 \mathrm{~min}$. and $2 \mu 1$ of this solution were injected into HP- 5 column.

2) Analysis after derivatization: Five mgs of illicit heroin sample were subjected to silylation with $150 \mu \mathrm{l}$ of N-methyl N-Tri Methyl Silyl Tri Fluro-Acetamide (MSTFA) in $1.2 \mathrm{ml}$ chloroform- pyridine $(5: 1 \mathrm{v} / \mathrm{v})$ for 10 minutes at $70{ }^{\circ} \mathrm{C}$. After 1 hour at room temperature, $2 \mu \mathrm{l}$ of this mixture were injected into HP-5 column and $1 \mu 1$ into DB-1 column.

\section{B) Neutral fraction}

For the determination of the neutral components of the illicit heroin sample, $30 \mathrm{mgs}$ of illicit heroin sample were dissolved in 
Citation: Badria FA, El-Neketi M, Saad HA (2018) Forensic Analysis of a Confiscated Illicit Heroin Sample. Curr Res Bioorg Org Chem: CRBOC-105. DOI: 10.29011/ CRBOC -105. 100005

$10 \mathrm{ml}$ of $0.5 \mathrm{~N}$ sulfuric acid, extracted by shaking with ether $(2 \mathrm{x}$, $5 \mathrm{ml}$ each), and centrifuged. The ethereal layer was dried over anhydrous sodium sulfate, evaporated to dryness using a rotary evaporator, the residue was dissolved in $75 \mu \mathrm{l}$ of chloroform and $2 \mu \mathrm{l}$ were injected directly into HP-5 column $[7,27,32]$. Silylation of the neutral fraction is not recommended as reported by [32,27]. The identification of the components was achieved by comparing the fragmentation pattern of the resulting mass spectra with those recorded in mass library spectral database and published data.

Operating conditions for GC $[2,3,7,33]$ (Table $1 \mathrm{~b}$ )

\begin{tabular}{|c|c|}
\hline Column (HP-5) & $\begin{array}{c}\text { Fused silica capillary column } \\
\text { (5\% phenyl) Methylsiloxane (HP-5) } \\
(0.25) \text { I.D, } 30 \mathrm{~m} \text {. length }\end{array}$ \\
\hline $\begin{array}{c}\text { Volume of } \\
\text { injected sample }\end{array}$ & $2 \mu \mathrm{l}$ \\
\hline $\begin{array}{c}\text { Injector } \\
\text { temperature }\end{array}$ & $250{ }^{\circ} \mathrm{C}$ \\
\hline $\begin{array}{c}\text { Column } \\
\text { temperature }\end{array}$ & $\begin{array}{c}\text { Start at } 150{ }^{\circ} \mathrm{C} \text {, Increase at } 6{ }^{\circ} \mathrm{C} / \text { minute to } 280 \\
{ }^{\circ} \mathrm{C} \text {, isothermal for } 10 \text { minute, Increase at } 9{ }^{\circ} \mathrm{C} / \\
\text { minute to } 300{ }^{\circ} \mathrm{C} \text {, isothermal for } 15 \text { minute, end } \\
\text { of program }\end{array}$ \\
\hline Column (DB-1) & $\begin{array}{l}\text { Fused silica capillary column } \\
\text { Dimethyl-polysiloxane (DB-1) } \\
\text { (0.32) I.D, } 30 \mathrm{~m} \text {. length }\end{array}$ \\
\hline $\begin{array}{c}\text { Volume of } \\
\text { injected sample }\end{array}$ & $1 \mu \mathrm{l}$ \\
\hline
\end{tabular}

\begin{tabular}{|c|c|}
\hline $\begin{array}{c}\text { Injector } \\
\text { temperature }\end{array}$ & $250{ }^{\circ} \mathrm{C}$ \\
\hline $\begin{array}{c}\text { Column } \\
\text { temperature }\end{array}$ & $\begin{array}{c}\text { Starts at } 150{ }^{\circ} \mathrm{C} \text {, Increases by } 9{ }^{\circ} \mathrm{C} / \text { minute to } 300 \\
{ }^{\circ} \mathrm{C} \text {, isothermal for } 10 \text { minute, end of program }\end{array}$ \\
\hline
\end{tabular}

\section{Results and discussion}

\section{Results}

\section{Physical Characters}

The physical characters of illicit heroin sample are summarized in (Table 2).

\begin{tabular}{|c|c|}
\hline Seizure shape & $\begin{array}{c}\text { Cylindrical pieces with rounded ends, wrapped } \\
\text { externally with green adhesive tape and } \\
\text { internally with another wrapping of yellowish- } \\
\text { white to light brown paper from which the } \\
\text { sample was obtained (chunks) measuring 0.5- } \\
1.5 \mathrm{~cm} \text { D. Figure (1 A\&B) }\end{array}$ \\
\hline Sample shape & $\begin{array}{c}\text { Small granular pieces (chunks) of about 0.5 to } \\
1.5 \text { cm in diameter (Figure 1 C) }\end{array}$ \\
\hline Sample weight & Dark brown to nearly black. \\
\hline Sample color & Rough \\
\hline Sample touch & Strong vinegar- like odor. \\
\hline Sample odor & Soluble in water producing brownish turbid \\
\hline s-Solution containing vegetable debris.
\end{tabular}

Table 2: Physical characters of illicit heroin sample.

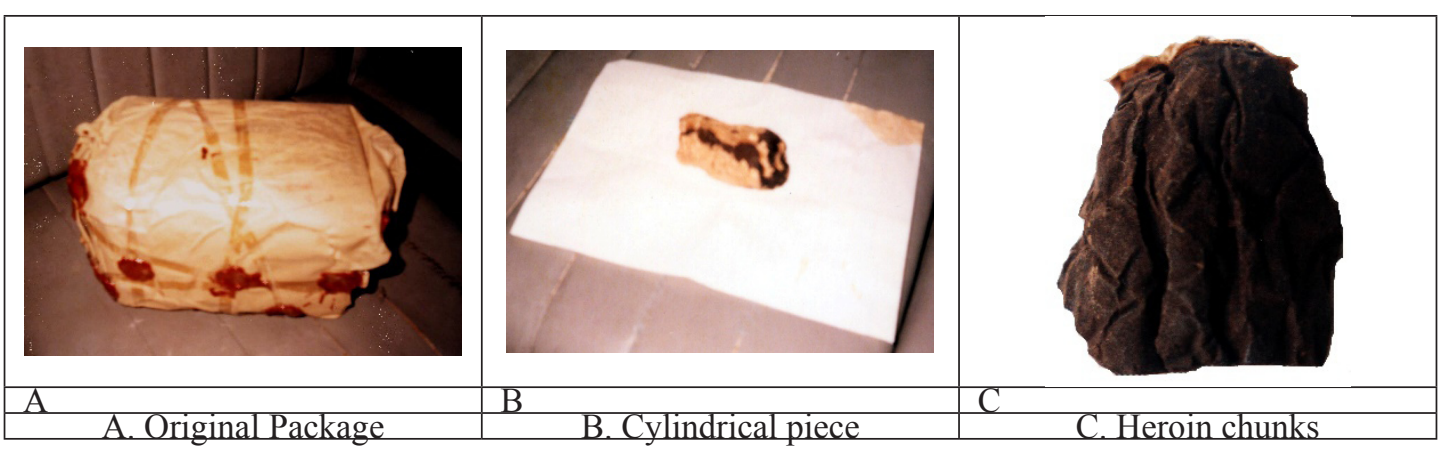

Figure 1: Confiscated heroin (seizure number 978/1988, Suez-Egypt).

\section{Microscopic Examination}

The following fragments (Figure 2) were detected

1-Abundant starch granules: simple, small rounded granules, with centric split or cleft hilum and faint concentric striations which are more visible in larger granules and measuring 11-12-15 $\mu$. (D.).

2-Long fibers: with thin non-lignified wall, wide lumen and acute apex measuring 225-456-825 $\mu$. (L.) and 15-24-30 $\mu$. (W.)

3-Short fibers: with thick non-lignified wall, narrow lumen and bluntly pointed apex measuring 122-211-237 $\mu$. (L.) and 17-20-23 $\mu$. (W.). 


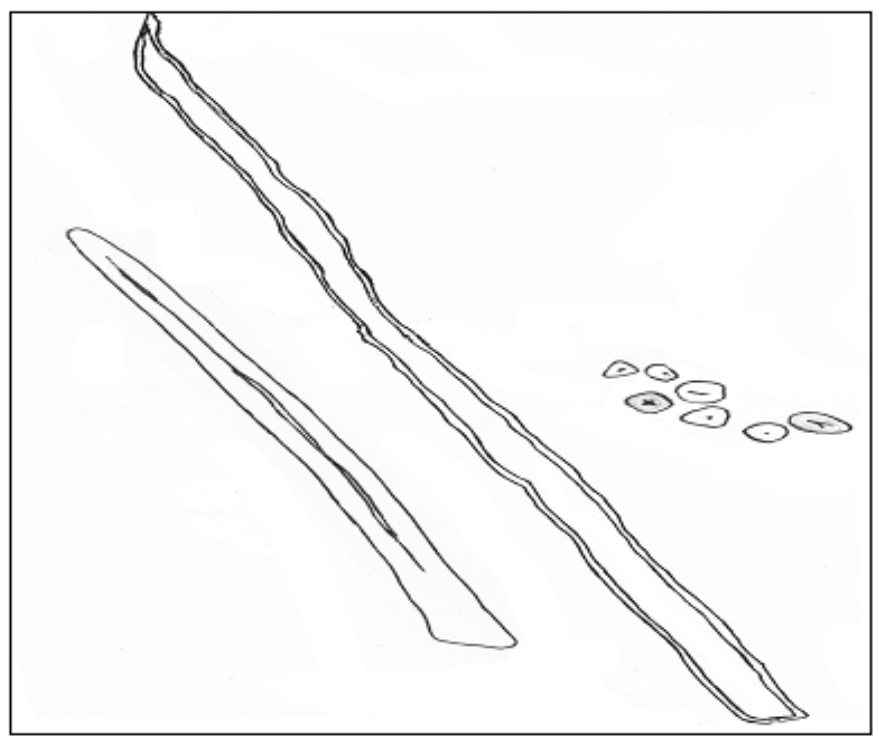

A-Starch granules $(X=284), B$ - L ong fiber with wide lumen $(X=169)$, C- Short fiber with narrowlumen $(\mathrm{X}=284)$.

Figure 2: Vegetable debris of the heroin sample.

\section{Color, Solubility and Precipitation Tests}

The results are shown in (Table 3)

\begin{tabular}{|c|c|c|}
\hline Test Name & Result & Indication \\
\hline \multicolumn{3}{|c|}{ Presumptive color tests } \\
\hline Marquis & A violet / reddish purple color & Presence of morphine, codeine or heroin. \\
\hline Froehde & A purple / green color & Presence of morphine, codeine or heroin. \\
\hline Meck & A blue / green color & Presence of morphine, codeine or heroin. \\
\hline Nitric acid & $\begin{array}{c}\text { A red-orange color was obtained at first } \\
\text { which was gradually changed to a bright green color }\end{array}$ & Presence of heroin. \\
\hline Oliver & A persistent pink to red color & Presence of heroin \\
\hline Murexide & $\begin{array}{l}\text { Purple color after exposure to ammonia } \\
\text { vapor. }\end{array}$ & $\begin{array}{l}\text { Presence of caffeine or other xanthine } \\
\text { alkaloids }\end{array}$ \\
\hline \multicolumn{3}{|c|}{ Solubility tests } \\
\hline Solubility in water & Soluble in water leaving vegetable debris & Presence of heroin salt \\
\hline Solubility in ethanol & $\begin{array}{l}\text { Soluble in ethanol, leaving vegetable } \\
\text { residue }\end{array}$ & Presence of starch \\
\hline \multicolumn{3}{|c|}{ Precipitation (Anion) test } \\
\hline Silver nitrate & No white precipitate & Absence of chloride ion. \\
\hline Barium chloride & White precipitate & Presence of sulfate ion. \\
\hline
\end{tabular}

Table 3: Results of the presumptive color and precipitation tests for the heroin sample. 
Citation: Badria FA, El-Neketi M, Saad HA (2018) Forensic Analysis of a Confiscated Illicit Heroin Sample. Curr Res Bioorg Org Chem: CRBOC-105. DOI: 10.29011/ CRBOC -105. 100005

\section{TLC}

The results are shown in (Table 4)

\begin{tabular}{|c|c|c|c|}
\hline \multirow{2}{*}{ Reference } & \multicolumn{2}{|c|}{$R_{\text {f } 100}$ Heroin sample } \\
\cline { 2 - 4 } & System A & 14.5 & $(+)$ \\
\hline Morphine & 16.9 & 28.9 & $(+)$ \\
\hline Codeine & 36.9 & 72.6 & $(+)$ \\
\hline Caffeine & 40.9 & 27.6 & $(+)$ \\
\hline $0^{6}$ monoacetylmorphine & 66.15 & 78.9 & $(+)$ \\
\hline Papaverine & 68.7 & 63.16 & $(+)$ \\
\hline Heroin & 69.2 & 55.3 & $(+)$ \\
\hline Acetylcodeine & 75.8 & $(+)$ & \\
\hline
\end{tabular}

Table 4: Results of TLC investigation of the heroin sample.

\section{GC/MS}

The GC chromatograms are shown in (Figures 3-6), while the results of GC/MS are cited in (Table 5,6) and illustrated in (Figures

7). The identified components of the heroin sample are listed in (Table 7) and shown in (Figures 8).

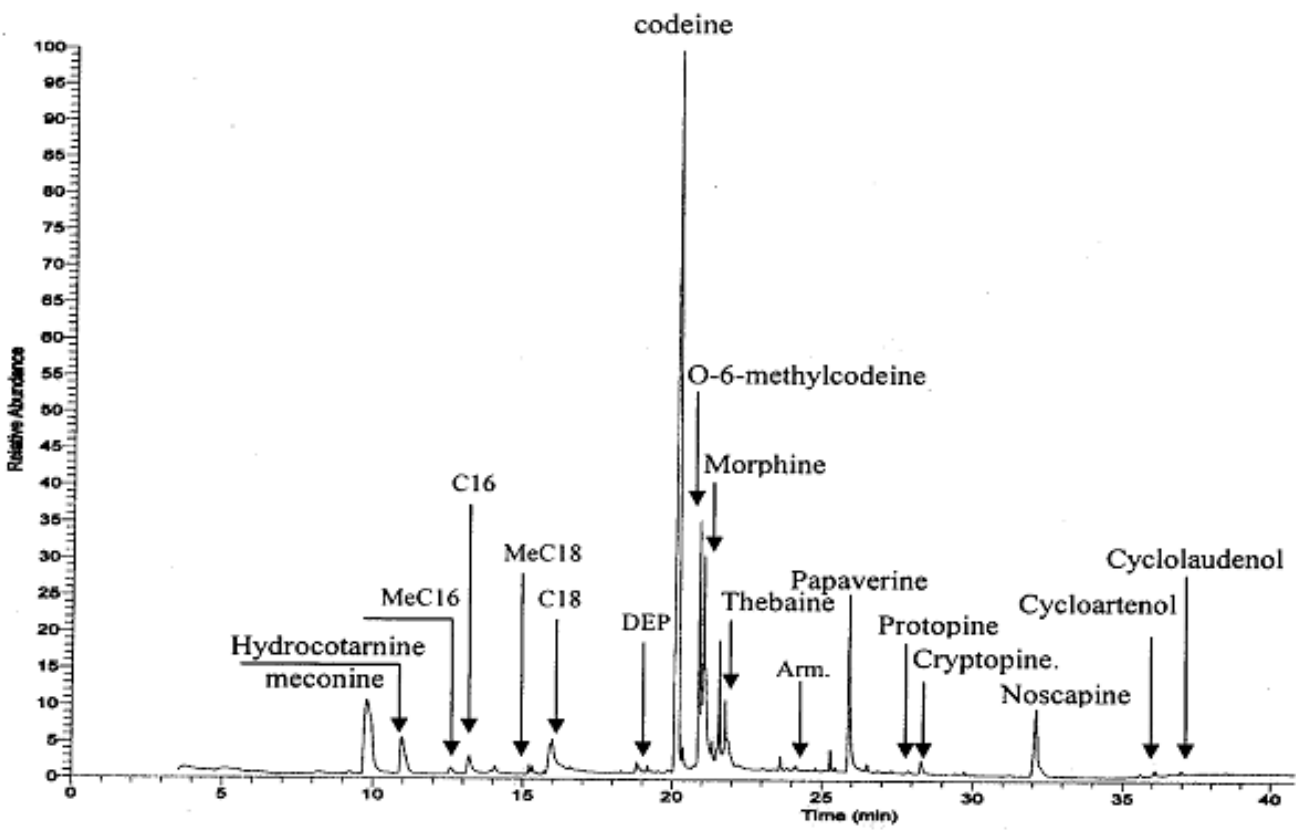

$\mathrm{MePh}$ Mephobarbital; MeOL: Methyl oleate (9-Octadecenoic acid methyl ester); MeSt: Methyl stearate (Octadecanoic acid methyl ester); DEP: 3,6-dimethoxy-4,5 epoxy-phenanthrene; Acl\&L.: May be a mixture of NA cetylnorlaudanosine and laudanosine.

Figure 3: GC of the non silylated heroin sample on (HP-5) column. 


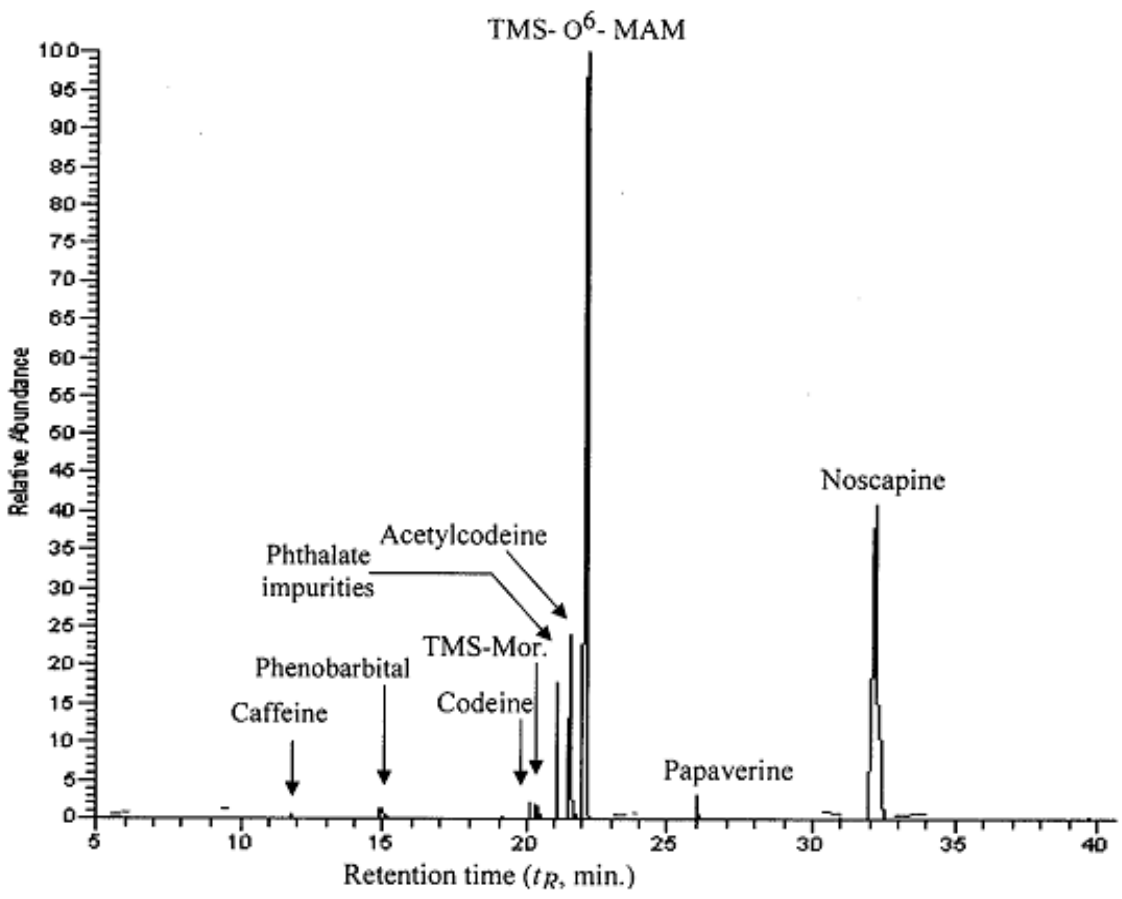

TMS-mor: Trimethylsilylmorphine; TMS-06-MAM: Trimethylsilyl- O6 monoacetyl-morphine.

Figure 4: GC of the silylated heroin sample (MSTAF) on (HP-5) column.

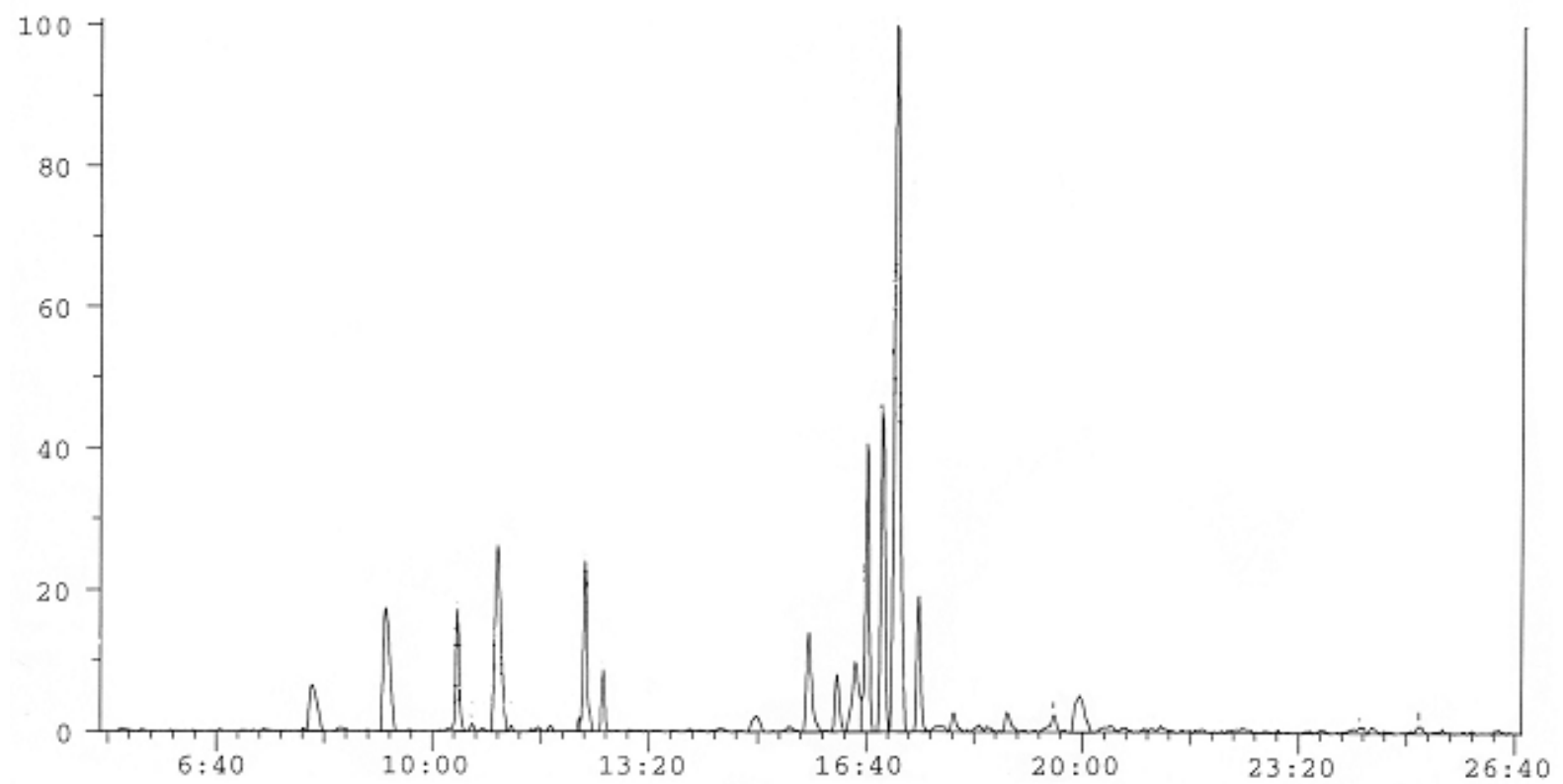

Me C16: Methyl palmitate (hexadecanoic acid methyl ester); MeOl: Methyl oleate (9-Octadecenoic acid methyl ester); MeSt: Methyl stearate (Octadecanoic acid methyl ester); DEP: 3,6 Dimethoxy- 4,5 epoxy-phenanthrene; TMS-Mor:: Timethylsilyl-morphine; TMS- O6-MAM: Timethylsilyl -O6 monoacetyl-morphine; AN: NAcetylanhydronornarceine

Figure 5: GC of the silylated heroin sample (MSTAF) on (DB-1) column. 
Citation: Badria FA, El-Neketi M, Saad HA (2018) Forensic Analysis of a Confiscated Illicit Heroin Sample. Curr Res Bioorg Org Chem: CRBOC-105. DOI: 10.29011/ CRBOC -105. 100005

\begin{tabular}{|c|c|c|c|c|c|c|c|c|c|c|}
\hline \multirow{3}{*}{ Component } & \multicolumn{3}{|c|}{$t_{R}, \min }$. & \multicolumn{3}{|c|}{ Relative\% } & \multirow{3}{*}{$\mathbb{M}^{+}$} & \multirow{3}{*}{$\begin{array}{l}\text { Base } \\
\text { Peak }\end{array}$} & \multirow{3}{*}{$\begin{array}{l}\text { Fragmentions } \\
\text { mbz (relative intensity) }\end{array}$} & \multirow{3}{*}{ Relerence } \\
\hline & \multirow{2}{*}{$\begin{array}{l}\text { Beiore } \\
\text { HP.5 } \\
\end{array}$} & \multicolumn{2}{|c|}{ After } & \multirow{2}{*}{$\begin{array}{c}\text { Before } \\
\text { HP.5 }\end{array}$} & \multicolumn{2}{|c|}{\begin{tabular}{|l|} 
After \\
\end{tabular}} & & & & \\
\hline & & HP.5 & DB.1 & & HP.5 & $\overline{D B-1}$ & & & & \\
\hline Nicotinamide & 4.75 & - & - & 1.39 & - & - & 122 & 122 & $78(63), 106(57), 51(10), 123(7)$ & 34 \\
\hline Isomeconine & - & - & 8.08 & - & - & 2.83 & 194 & 194 & $165(87), 147(56), 50(35), 63(33), 176(22), 77(27)$ & \\
\hline Meconine & 9.82 & - & - & 8.42 & - & - & $194(97)$ & 165 & $147(77), 176(47), 188(30), 199(30), 77(27)$ & 32 \\
\hline Hydrocotamine & 10.96 & - & - & 1.94 & - & - & $221(6)$ & 220 & $178(74), 205(21), 165(20), 177(19)$ & 35,36 \\
\hline Caffeine & 11.43 & 11.8 & 9.15 & 7,13 & 0.17 & 6.34 & 194 & 194 & $109(46), 55(25), 67(20), 82(17), 193(14), 165(7), 137(6)$ & 34 \\
\hline Methyl palmitate & - & - & 10.2 & - & - & 3.44 & $270(17)$ & 74 & $87(70), 143(21), 55(20), 227(15)$ & 37 \\
\hline $\begin{array}{l}\text { Methyl phenobaritital } \\
\text { (Mephobaritital) }\end{array}$ & 12.67 & - & - & 1.03 & - & - & $246(10)$ & 218 & $117(33), 46(20), 118(16), 114(15)$ & 34 \\
\hline Phenobarbital & 14.03 & 14.9 & 10.6 & 5.02 & 0.77 & 8.85 & $232(20)$ & 204 & $117(27), 161(16), 146(12), 205(11), 77(10)$ & 34 \\
\hline Methyl oleate & 15.33 & - & 12.2 & 0.08 & - & 4.9 & $296(10)$ & 55 & $83(39), 69(36), 74(34), 264(32), 96(24), 222(13), 110(11)$ & 34,36 \\
\hline Methyl stearate & 15.73 & - & 12.4 & 0.09 & - & 1.55 & $298(21)$ & 74 & $87(70), 55(25), 143(28), 75(24), 255(16), 1999(11)$ & 34,37 \\
\hline DEP & 18.82 & - & 14.6 & 0.69 & - & 0.98 & $252(85)$ & 237 & $194(21), 138(15)$ & 38 \\
\hline Codeine & 20.10 & 20.1 & 15.5 & 6.41 & 0.37 & 4.03 & 299 & 299 & $162(36), 229(22), 300(21), 124(19), 282(8), 242(10)$ & 39 \\
\hline TMS. Morphine & - & 20.3 & 16.1 & - & 0.36 & 1.96 & 429(52) & 73 & $236(28), 146(18), 196(17), 444(16)$ & 2 \\
\hline Morphine & 21.09 & - & - & 12.79 & - & - & 285 & 285 & $162(35), 215(23), 286(18), 124(16), 288(15), 228(7)$ & 34,40 \\
\hline Acetylcodeine & 21.69 & 21.6 & 16.6 & 7.72 & 5.38 & 10.6 & 341 & 341 & $282(55), 229(33), 204(22), 124(15), 59(15) 162(14), 115(12)$ & 2 \\
\hline $0^{6}$.MAM & 22.06 & - & 17.1 & 27.20 & - & 30.7 & 327 & 327 & $\begin{array}{l}288(14), 215(27), 146(20), 328(18), 162(15), 204(14), 124(13), 81 \\
(12)\end{array}$ & 26 \\
\hline TMS. $0^{6}$ MAM & - & 22.1 & 17.3 & - & 41.4 & 4.0 & 399 & 399 & $73(72), 340(61), 287(40), 204(33), 324(28)$ & \\
\hline Heroin & 23.22 & - & 18.0 & 0.54 & - & 0.72 & $369(56)$ & 327 & $268(52), 310(45), 204(36), 215(32), 328(20), 81(19), 162(18)$ & 2 \\
\hline Papaverine & 25.91 & 26.0 & 19.6 & 2.88 & 0.99 & 2.8 & 338 & 338 & $324(97), 339(90), 308(20), 325(15), 154(14)$ & \\
\hline Cryptopine & 28.33 & - & - & 0.07 & - & - & $369(4)$ & 148 & $179(20), 169(10), 190(7), 91(6)$ & 41 \\
\hline
\end{tabular}

Table 5: Results of GC-MS of identified components of the heroin sample before and after silylation (MSTAF) using HP-5 and DB-1 columns.

\begin{tabular}{|c|c|c|c|c|c|c|c|c|c|c|}
\hline \multirow{3}{*}{ Component } & \multicolumn{3}{|c|}{$t_{B}$ min } & \multicolumn{3}{|c|}{ Relative\% } & \multirow{3}{*}[\mathbb{M}]{$^{+}$} & \multirow{3}{*}{$\begin{array}{l}\text { Base } \\
\text { Peak }\end{array}$} & \multirow{3}{*}{$\begin{array}{c}\text { Fragmentions } \\
m b \text { (relative intensity) }\end{array}$} & \multirow{3}{*}{ References } \\
\hline & \multirow{2}{*}{$\begin{array}{l}\text { Belone } \\
\text { HP.5 }\end{array}$} & \multicolumn{2}{|c|}{\begin{tabular}{|l|l|} 
Alter \\
\end{tabular}} & \multirow{2}{*}{$\begin{array}{l}\text { Belont } \\
\text { HPS. }\end{array}$} & \multicolumn{2}{|c|}{ After } & & & & \\
\hline & & HPS.S & DB-1 & & HPS & $\mathrm{DBA}$ & & & & \\
\hline $\begin{array}{l}\text { Mixture of Laudanosine \& N- } \\
\text { Acetylnorlaudanosine } \\
\text { Laudanosine } \\
\text { N-Acetynorlaudanosine }\end{array}$ & 29.66 & - & - & 0.06 & - & - & $\begin{array}{l}357(3) \\
385\end{array}$ & $\begin{array}{l}192 \\
234\end{array}$ & $235(12), 151(10), 107(6)$ & $\begin{array}{l}42 \\
32\end{array}$ \\
\hline Noscapine & 32.28 & 322 & 25.3 & 13.7 & 34.7 & Trace & 413 & 220 & $21(14), 205(11)$ & 2 \\
\hline May be: N-Acetylanhydronormarceine & - & - & 25.5 & - & - & Trace & 455 & 193 & & 32 \\
\hline
\end{tabular}

Methyl palmitate: (hexadecanoic acid methyl ester); Methyl oleate: (9-0ctadecenoic acid methyl ester); Methyl stearate (Octadecanoic acid methyl ester); DEP: 3,6 Dimethoxy- 4,5 epoxyphenanthrene; TMS- Morphine: trimethylsilyl morphine; $0^{6}$ MAM: $0^{6}$ monoacetylmorphine; TMS- $0^{6}$ MAM: Trimethylsilyl-06 monoacetylmorphine

Table (5): Cont. 
Citation: Badria FA, El-Neketi M, Saad HA (2018) Forensic Analysis of a Confiscated Illicit Heroin Sample. Curr Res Bioorg Org Chem: CRBOC-105. DOI: 10.29011/ CRBOC -105. 100005

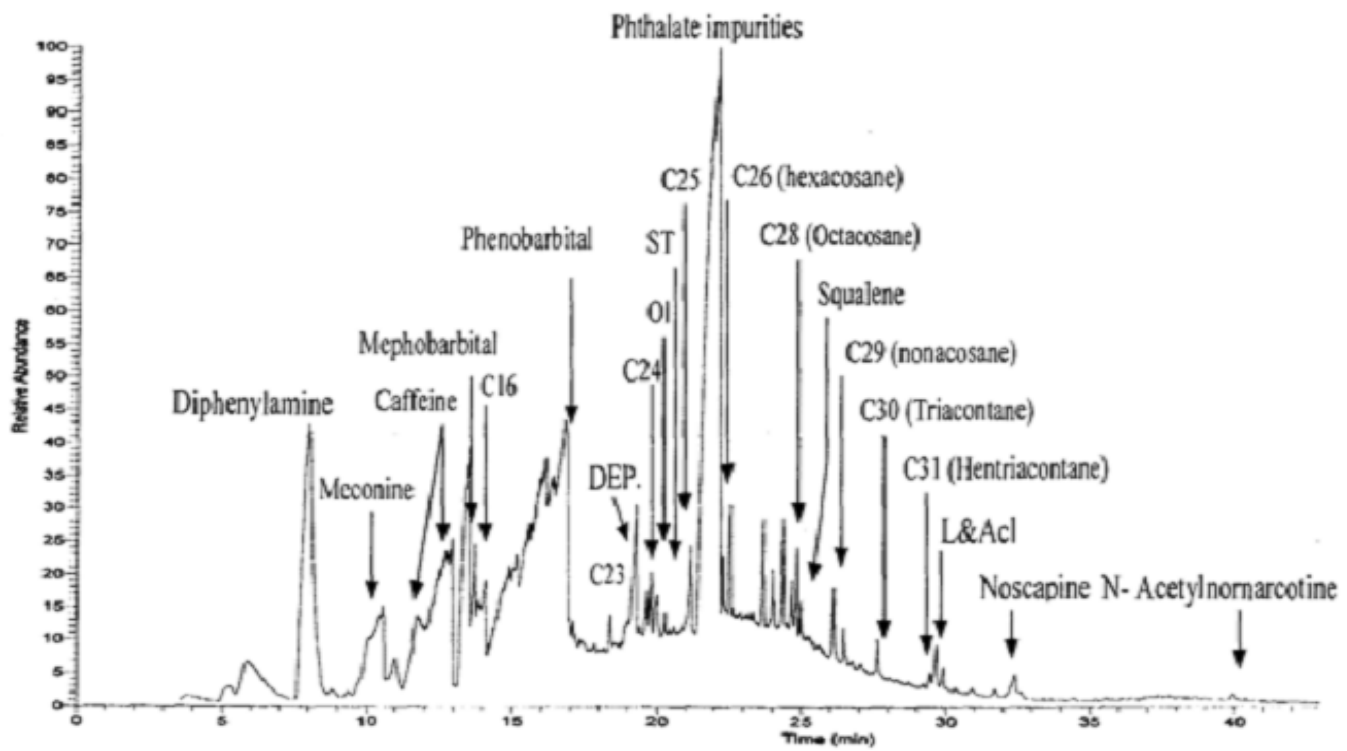

C16: Palmitic acid (Hexadecanoic acid); C23: n- Tricosane; DEP: 3,6 Dimethoxy- 4,5 epoxyphenan-threne; C24: nTetracosane; O1: Oleoamide (9-Octadecenamide); ST: Ste aramide (Octadecanamide); C25: Pentacosane; L\&Acl: mixture of N-A cetylnorlaudanosine and laudanosine

Figure 6: GC of non silylated neutral fraction of the heroin sample using HP-5 column.

\begin{tabular}{|c|c|c|c|c|c|c|}
\hline Component & $t_{R}$ min. & Relative \% & {$\left[M^{+}\right.$} & $\begin{array}{l}\text { Base } \\
\text { Peak }\end{array}$ & $\begin{array}{c}\text { Fragment ions } \\
m / 2 \text { (relative intensity) }\end{array}$ & Reforence \\
\hline Diphenylamine & 8.02 & 11.1 & 169 & 169 & $168(60), 167(34), 83(23), 170(15), 51(10), 77(10)$ & 34 \\
\hline Meconine & 10.5 & 4.24 & $194(97)$ & 165 & $147(77), 176(47), 118(30), 119(30), 77(27)$ & 32 \\
\hline Caffeine & 12.9 & 9.84 & 194 & 194 & $109(46), 55(25), 67(20), 82(17), 193(14), 165(7), 137(6)$ & \multirow[t]{2}{*}{34} \\
\hline Methl phenobarbital (Mephobarbital) & 13.7 & 2.81 & $246(10)$ & 218 & $117(33), 146(20), 118(16), 114(15)$ & \\
\hline Palmitic acid (Hexadecanoic acid) & 14.1 & 1.21 & $256(55)$ & 73 & $\begin{array}{l}60(82), 57(76), 55(60), 129(55), 72(46), 69(36), 85(34), 83(32), \\
213(31), 97(30), 157(25)\end{array}$ & 34,36 \\
\hline Phenobarbital & 16.8 & 12.2 & $232(20)$ & 204 & $117(27), 161(16), 146(12), 205(11), 77(10)$ & \multirow{2}{*}{34} \\
\hline n-Tricosane & 18.4 & 0.42 & 324 & 57 & $71(77), 85(56), 99(21), 113(15), 127(10), 141(7)$ & \\
\hline DEP & 19.3 & 2.68 & $252(85)$ & 237 & $194(21), 138(15)$ & 38 \\
\hline n- Tetracosane & 19.8 & 0.59 & $338(6)$ & 57 & $71(77), 85(56), 99(21), 113(15), 127(11), 141(7), 155(5)$ & 34 \\
\hline Oleoamide (9-0ctadecenamide) & 19.9 & 0.61 & $281(6)$ & 59 & $72(67), 126(16), 83(15), 97(12), 112(11)$ & 36 \\
\hline Stearamide (Octadecanamide) & 20.3 & 0.19 & $283(5)$ & 59 & $72(44), 58(15), 128(12), 58(10), 240(7), 86(6)$ & \multirow{2}{*}{34} \\
\hline Pentacosane & 21.2 & 1.23 & $352(7)$ & 57 & $71(76), 85(59), 99(21), 113(16), 127(10), 141(7)$ & \\
\hline Hexacosane & 22.5 & 0.07 & $366(7)$ & 57 & $71(76), 85(60), 99(24), 113(16), 239(11)$ & \multirow[t]{6}{*}{34} \\
\hline Octacosane & 24.8 & 0.7 & $394(5)$ & 57 & $71(78), 85(60), 99(25), 113(16), 127(11), 141(7)$ & \\
\hline Squalene & 25.0 & 0.22 & $410(1)$ & 69 & $81(55), 149(41), 137(16), 121(15)$ & \\
\hline Nonacosane & 26.1 & 0.74 & $408(6)$ & 57 & $71(80), 85(62), 99(25), 113(17), 338(15)$ & \\
\hline Triacontane & 27.6 & 0.4 & $422(2)$ & 57 & $71(80), 85(61), 99(25), 113(16), 127(12), 141(10)$ & \\
\hline \multirow{4}{*}{$\begin{array}{l}\text { Hentriacontane } \\
\text { mixture of : laudanosine and } \\
\text { N-Acetylnorlaudanosine } \\
\text { Laudanosine } \\
\text { N-Acetylnorlauanosine }\end{array}$} & 29.5 & 0.18 & 436 & 57 & $71(76), 85(60), 99(25), 113(17), 127(12), 141(10)$ & \\
\hline & \multirow[t]{3}{*}{29.9} & \multirow[t]{3}{*}{0.28} & & & & \\
\hline & & & $357(3)$ & 192 & & 42 \\
\hline & & & 385 & 234 & $235(12), 151(10), 107(6)$ & 32 \\
\hline Noscapine & 32.4 & 0.51 & 413 & 220 & $221(14), 205(11)$ & 2 \\
\hline N-Acetylnonarcotine & 39.9 & 0.17 & 411 & 248 & $206(69), 191(15), 207(10), 133(6)$ & 32,36 \\
\hline
\end{tabular}

DEP: 3,6 Dimethoxy- 4,5 epoxyphenanthrene; (-) Not detected

Table 6: Results of GC-MS of identified components of non silylated neutral fraction of the heroin sample using HP-5 column. 


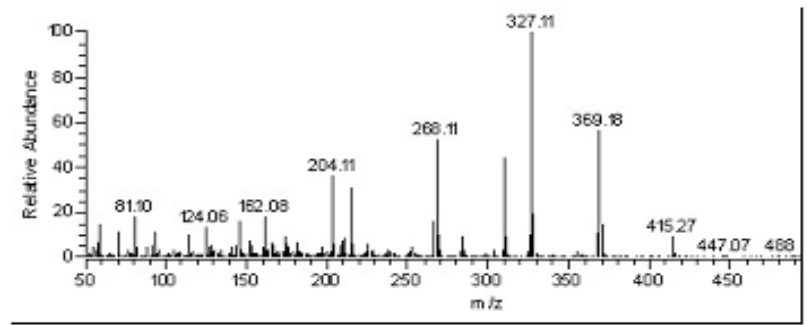

EI-MS spectrum of her oin (diacetylmorphine)

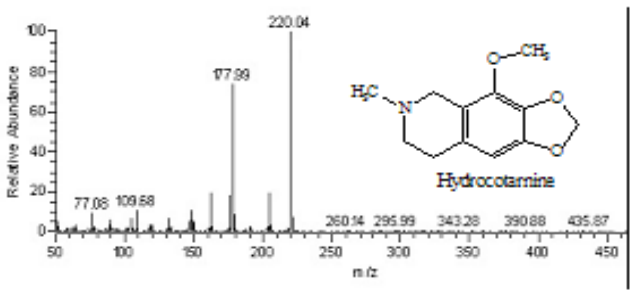

EI-MS spectrum of hydrocotarnine

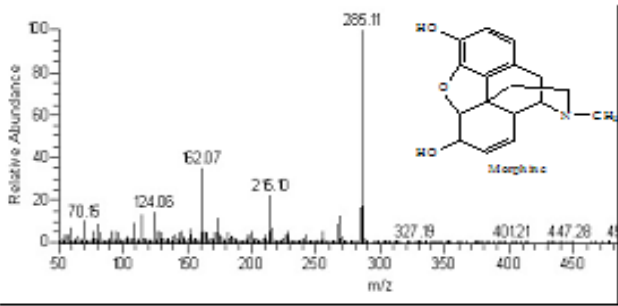

EI-MS spectrum of morphine

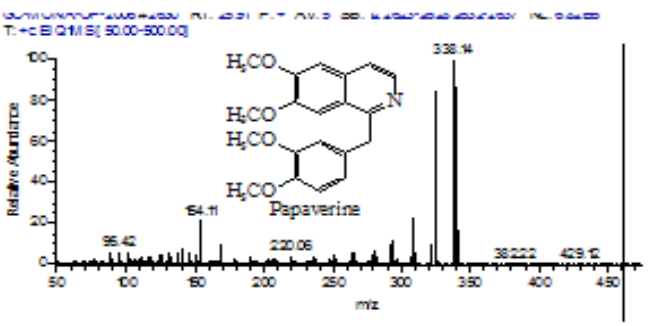

EI-MS spectrum of papaverine

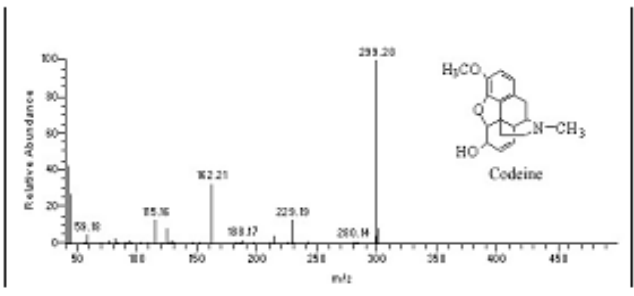

EI-MS spectrum of codeine

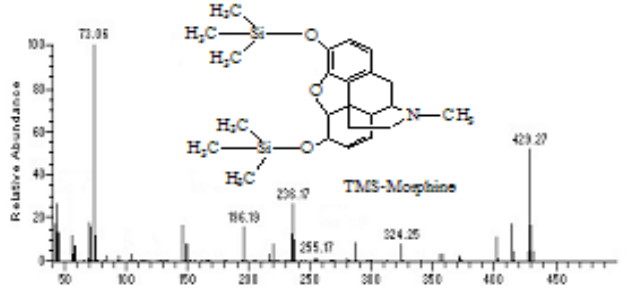

EI-MS spectrum of TMS-morphine

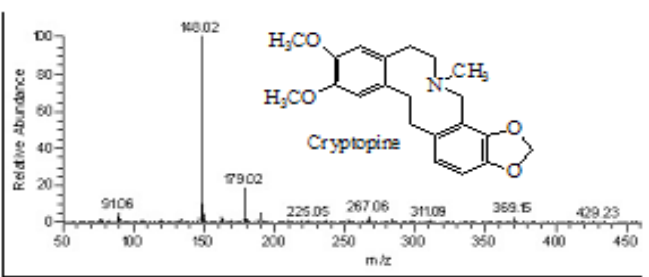

EI-MS spectrum of cryptopine

Figure (7): EI-MS spectrum of the detected components in heroin samples

Figure 7: EI-MS spectrum of the detected components in heroin samples. 


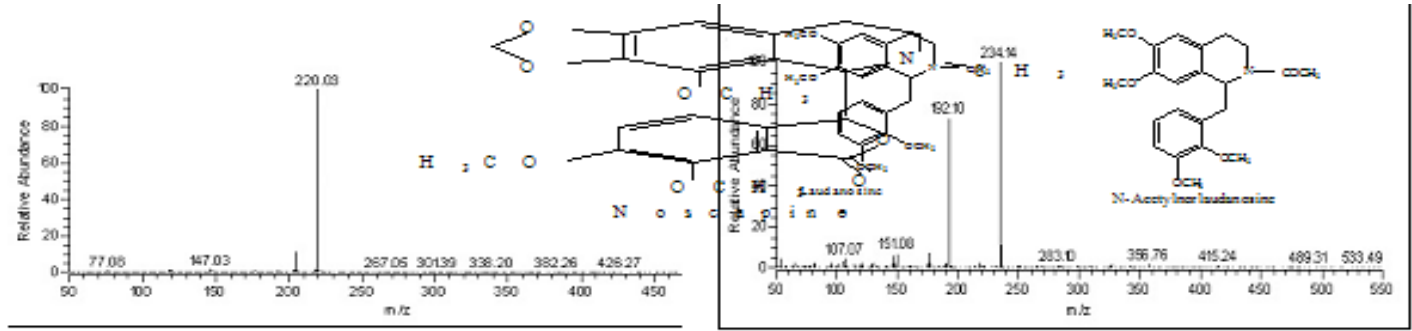

EI-MS spectrum of Noscapine

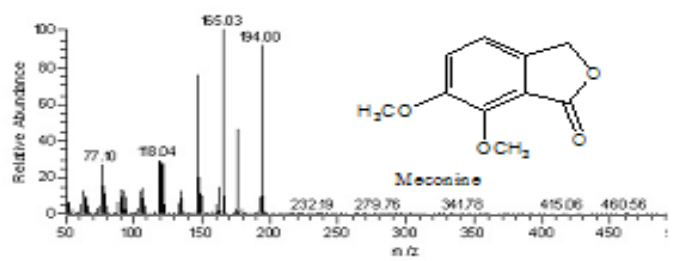

EI-MS spectrum of Meconine

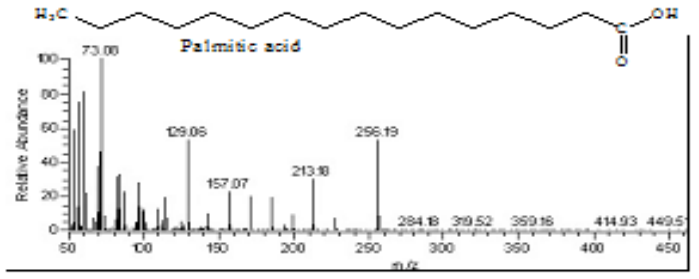

EI-MS spectrum of Palmitic acid (hexadecanoic acid)

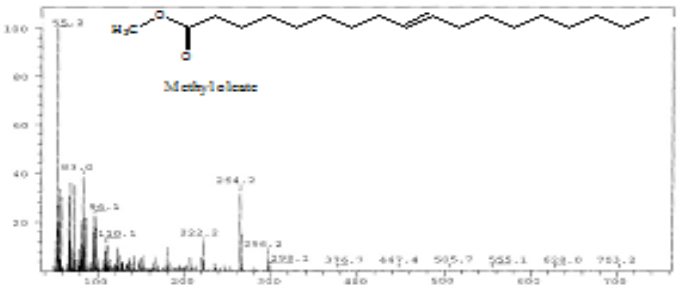

EI-MS spectra of methyl oleate (9-octadecenoic acid methyl ester)

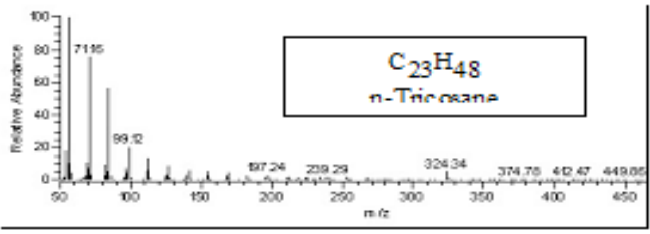

EI-MS spectrum of mixture of L audanosi ne and NAcetylnorlaudanosine

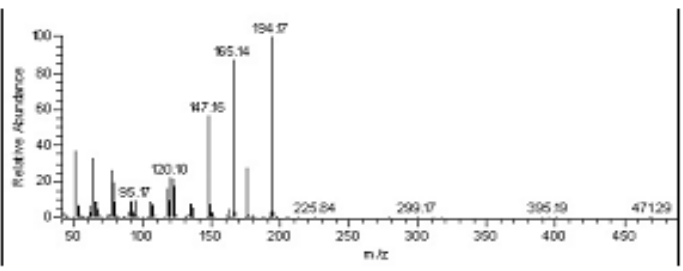

EI-MS spectrum of iso-meconine

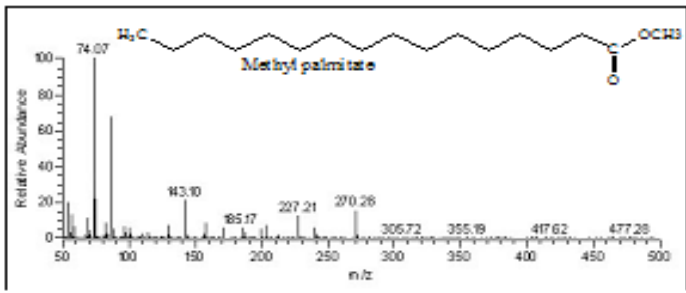

EI-MS spectrum of methyl palmitate (hexadecanoic acid methyl ester)

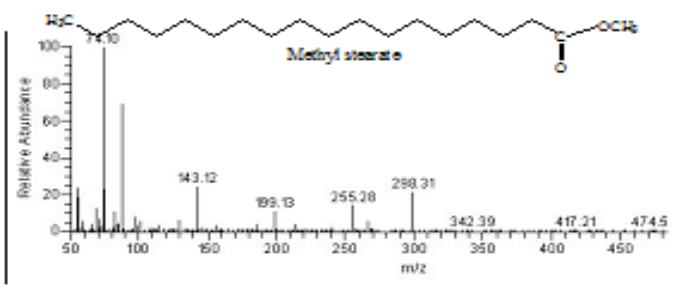

EI-MS spectrum of methyl stearate (Octadecanoic acid methyl ester)

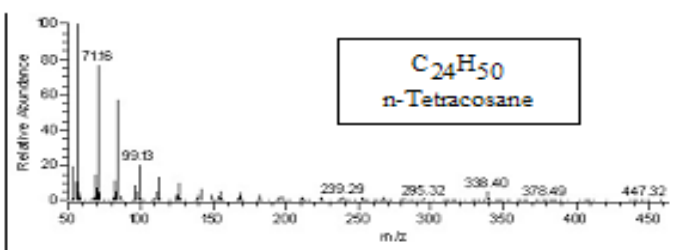

Figure (7): Cont. 
Citation: Badria FA, El-Neketi M, Saad HA (2018) Forensic Analysis of a Confiscated Illicit Heroin Sample. Curr Res Bioorg Org Chem: CRBOC-105. DOI: 10.29011/ CRBOC -105. 100005
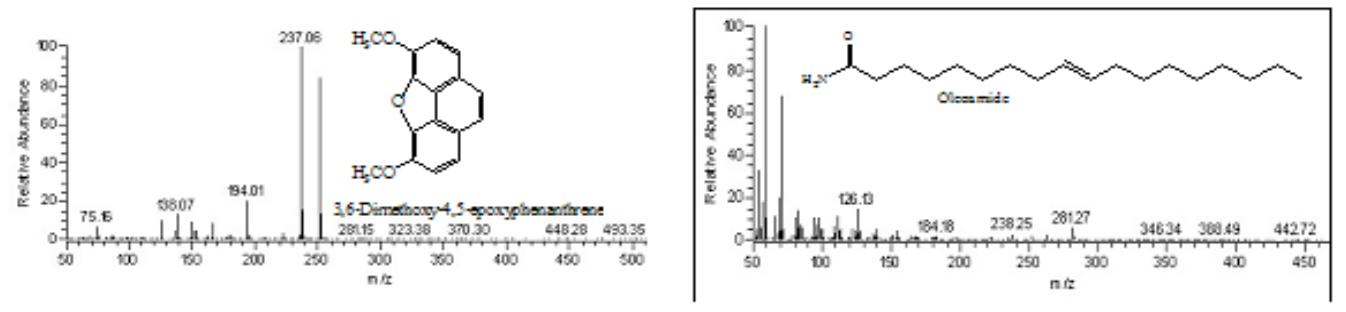

EI-MS spectrum of 3,6 Dimethoxy- 4,5 epoxy- Mass spectra of Oleoamide (9-Octadecenamide) phenanthrene
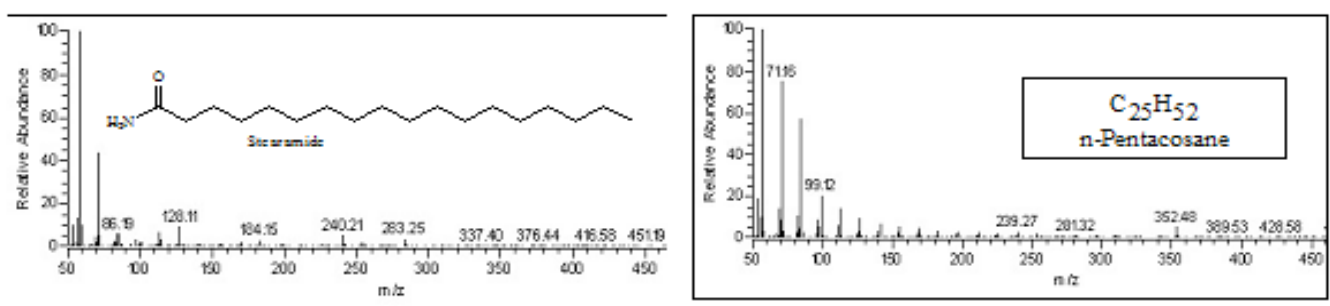

Mass spectra of Stearamide (Octadecanamide)

Mass spectra of n-Pentacosane
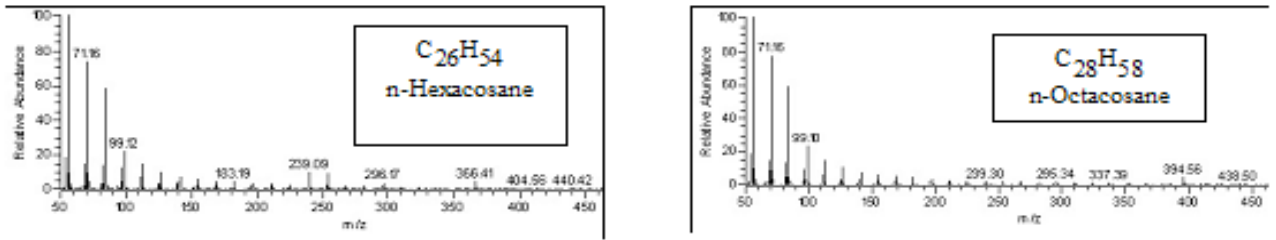

Mass spectra of n-Hexacosane

Mass spectra of n-Octaco sane
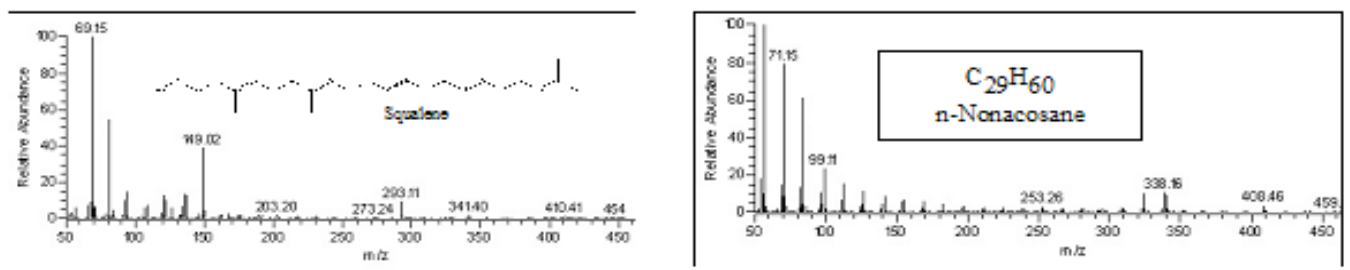

Mass spectra of squalene

Mass spectra of Nonacosane
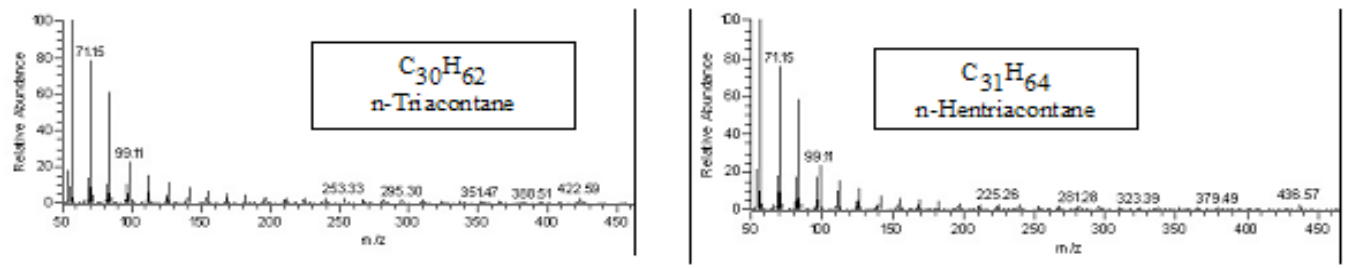

Mass spectra of Triacontane

Mass spectra of n-Hentriacontane

Figure (7): Cont. 


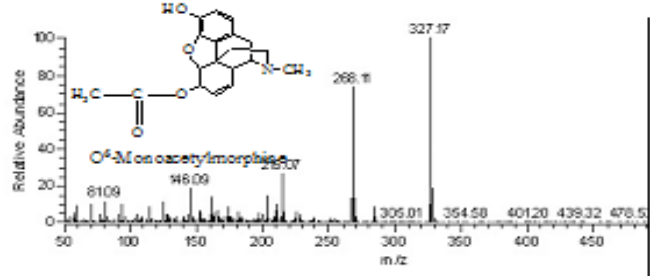

EI-MS spectrum of $\mathrm{O}^{6}$-monoacetyl-morphine (O6-MAM)

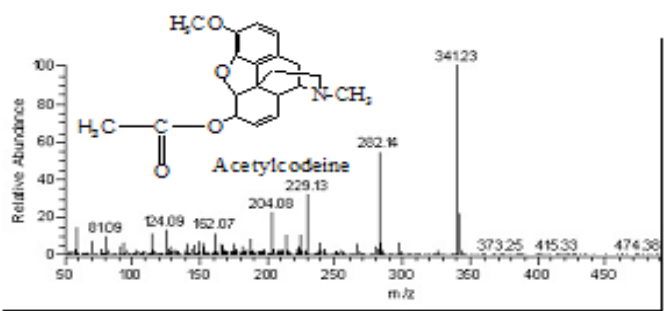

EI-MS spectrum of Acetylcodeine

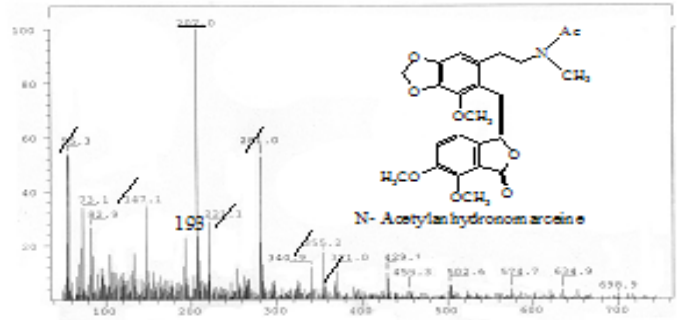

EI-MS spectrum of N-A cetylanhydronornarceine

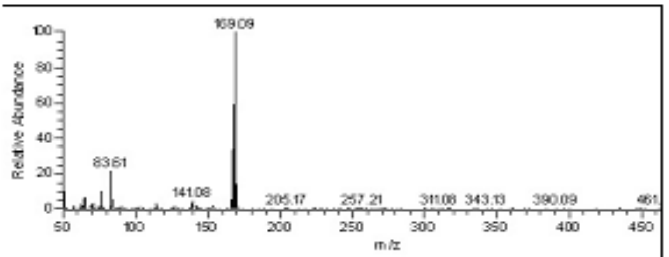

Mass spectra of Diphenylamine.

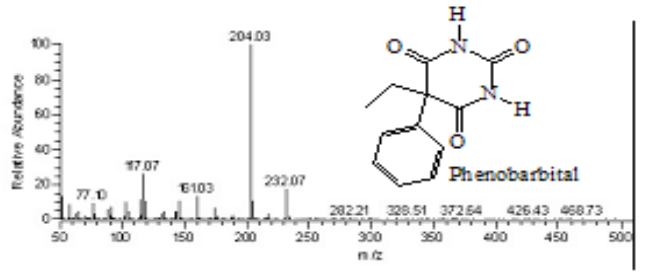

Mass spectra of Phenobarbital

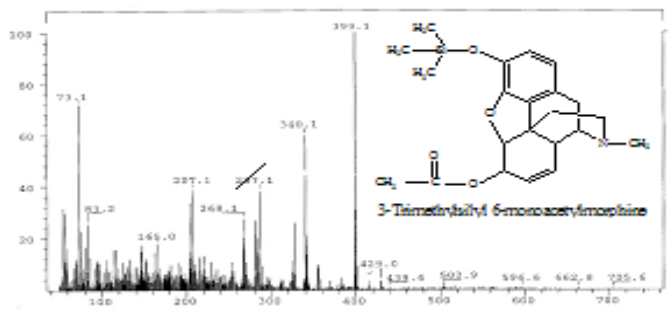

EI-MS spectrum of 3-Timethylsilyl-O 6 monoacetylmorphine (TMS-O 6 -MAM).

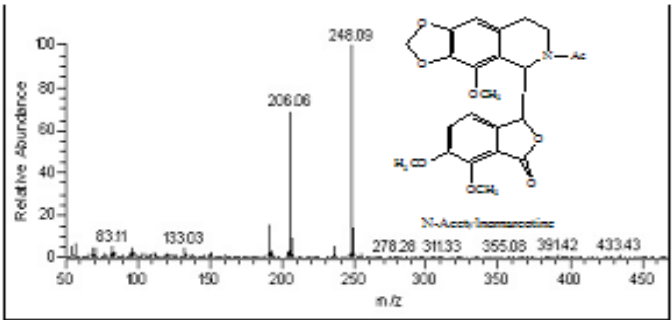

EI-MS spectrum of $\mathrm{N}$ - A cetylnornarcotine

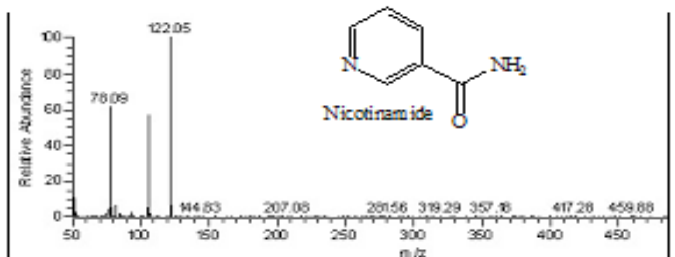

Mass spectra of Nicotinamide

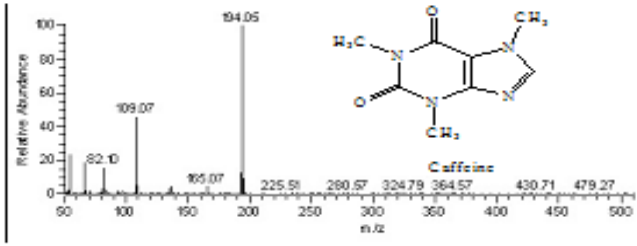

Mass spectra of Caffeine

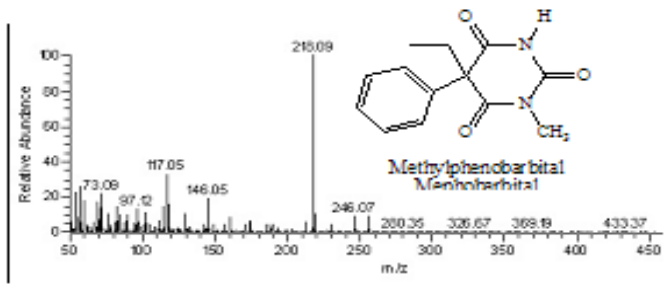

Mass spectra of Mephobarbital

Figure 7: Cont. 
Citation: Badria FA, El-Neketi M, Saad HA (2018) Forensic Analysis of a Confiscated Illicit Heroin Sample. Curr Res Bioorg Org Chem: CRBOC-105. DOI: 10.29011/ CRBOC -105. 100005

\begin{tabular}{|c|c|c|c|c|}
\hline Component & Molecular formula & Molecular weight & Mean relative $\%$ & Notes \\
\hline Heroin & $\mathrm{C}_{21} \mathrm{H}_{23} \mathrm{NO}_{5}$ & 369 & 0.6 & \\
\hline \multicolumn{5}{|c|}{ Natural impurities from opium: } \\
\hline Hydrocotarnine & $\mathrm{C}_{12} \mathrm{H}_{15} \mathrm{NO}_{3}$ & 221 & 1.94 & \\
\hline Codeine & $\mathrm{C}_{18} \mathrm{H}_{21} \mathrm{NO}_{3}$ & 299 & 3.6 & \\
\hline Morphine & $\mathrm{C}_{17} \mathrm{H}_{19} \mathrm{NO}_{3}$ & 285 & 5 & \\
\hline Papaverine & $\mathrm{C}_{20} \mathrm{H}_{21} \mathrm{NO}_{4}$ & 339 & 2.2 & \\
\hline Cryptopine & $\mathrm{C}_{21} \mathrm{H}_{23} \mathrm{NO}_{5}$ & 369 & 0.07 & \\
\hline Noscapine & $\mathrm{C}_{22} \mathrm{H}_{23} \mathrm{NO}_{7}$ & 413 & 13.7 & \\
\hline laudanosine & $\mathrm{C}_{21} \mathrm{H}_{27} \mathrm{NO}_{4}$ & 357 & Trace & \\
\hline Meconine & $\mathrm{C}_{10} \mathrm{H}_{10} \mathrm{O}_{4}$ & 194 & 5.7 & \\
\hline $\begin{array}{c}\text { Methyl palmitate (Hexadecanoic acid methyl } \\
\text { ester) }\end{array}$ & $\mathrm{C}_{17} \mathrm{H}_{34} \mathrm{O}_{2}$ & 270 & 3.4 & \\
\hline Palmitic acid (Hexadecanoic acid) & $\mathrm{C}_{16} \mathrm{H}_{32} \mathrm{O}_{2}$ & 256 & Trace & \\
\hline $\begin{array}{c}\text { Methyl oleate (9-Octadecenoic acid methyl } \\
\text { ester) }\end{array}$ & $\mathrm{C}_{19} \mathrm{H}_{36} \mathrm{O}_{2}$ & 296 & 2.5 & \\
\hline $\begin{array}{c}\text { Methyl stearate (Octadecanoic acid methyl } \\
\text { ester) }\end{array}$ & $\mathrm{C}_{19} \mathrm{H}_{38} \mathrm{O}_{2}$ & 298 & 0.8 & \\
\hline n-Tricosane & $\mathrm{C}_{23} \mathrm{H}_{48}$ & 324 & Trace & \\
\hline 3,6 Dimethoxy- 4,5 epoxy-phenanthrene & $\mathrm{C}_{16} \mathrm{H}_{12} \mathrm{O}_{3}$ & 252 & 0.8 & $\begin{array}{c}\text { Thebaine }+\mathrm{Ac}_{2} \mathrm{O} \\
\text { Decomposition } \\
\text { product }\end{array}$ \\
\hline n- Tetracosane & $\mathrm{C}_{24} \mathrm{H}_{50}$ & 338 & Trace & \\
\hline Oleoamide (9-Octadecenamide) & $\mathrm{C}_{18} \mathrm{H}_{35} \mathrm{NO}$ & 281 & Trace & \\
\hline Stearamide (Octadecanamide) & $\mathrm{C}_{18} \mathrm{H}_{37} \mathrm{NO}$ & 283 & Trace & \\
\hline Pentacosane & $\mathrm{C}_{22} \mathrm{H}_{52}$ & 352 & Trace & \\
\hline Hexacosane & $\mathrm{C}_{26} \mathrm{H}_{54}$ & 366 & Trace & \\
\hline Octacosane & $\mathrm{C}_{28} \mathrm{H}_{58}$ & 394 & Trace & \\
\hline Squalene & $\mathrm{C}_{30} \mathrm{H}_{50}$ & 410 & Trace & \\
\hline Nonacosane & $\mathrm{C} 2{ }_{9} \mathrm{H}_{60}$ & 408 & Trace & \\
\hline Triacontane & $\mathrm{C}_{30} \mathrm{H}_{62}$ & 422 & Trace & \\
\hline Hentriacontane & $\mathrm{C} 3{ }_{1} \mathrm{H}_{64}$ & 436 & Trace & \\
\hline
\end{tabular}


Citation: Badria FA, El-Neketi M, Saad HA (2018) Forensic Analysis of a Confiscated Illicit Heroin Sample. Curr Res Bioorg Org Chem: CRBOC-105. DOI: 10.29011/ CRBOC -105. 100005

\begin{tabular}{|c|c|c|c|c|}
\hline O6 - Monoacetylmorphine & $\mathrm{C}_{19} \mathrm{H}_{21} \mathrm{NO}_{4}$ & 327 & 34.4 & $\begin{array}{l}\text { Present in high } \\
\text { concentration due to } \\
\text { hydrolysis of heroin } \\
\text { (Small quantities } \\
\text { from morphine }+ \\
\mathrm{Ac}_{2} \mathrm{O} \text { ) }\end{array}$ \\
\hline Acetylcodeine & $\mathrm{C}_{20} \mathrm{H}_{23} \mathrm{NO}_{4}$ & 341 & 7.9 & \\
\hline N- Acetylnorlaudanosine & $\mathrm{C}_{22} \mathrm{H}_{27} \mathrm{NO}_{5}$ & 234 & 0.06 & \\
\hline N-Acetylnornarcotine & $\mathrm{C}_{23} \mathrm{H}_{23} \mathrm{NO}_{8}$ & 441 & Trace & $\begin{array}{c}\text { Noscapine } \\
+\mathrm{O}_{2}+\mathrm{Ac}_{2} \mathrm{O} \\
\text { Decomposition } \\
\text { product }\end{array}$ \\
\hline N-Acetylanhydronornarceine & $\mathrm{C}_{24} \mathrm{H}_{25} \mathrm{NO}_{8}$ & 455 & Traces & $\begin{array}{c}\text { Noscapine }+\mathrm{Ac}_{2} \mathrm{O} \\
\text { Decomposition } \\
\text { product }\end{array}$ \\
\hline \multicolumn{5}{|c|}{ Adulterants: } \\
\hline Nicotinamide & $\mathrm{C}_{6} \mathrm{H}_{6} \mathrm{~N}_{2} \mathrm{O}$ & 122 & 1.39 & \\
\hline Diphenylamine & $\mathrm{C}_{12} \mathrm{H}_{11} \mathrm{~N}$ & 169 & Trace & \\
\hline
\end{tabular}

Table 7: Identified components of the illicit heroin sample.

\section{Heroin}

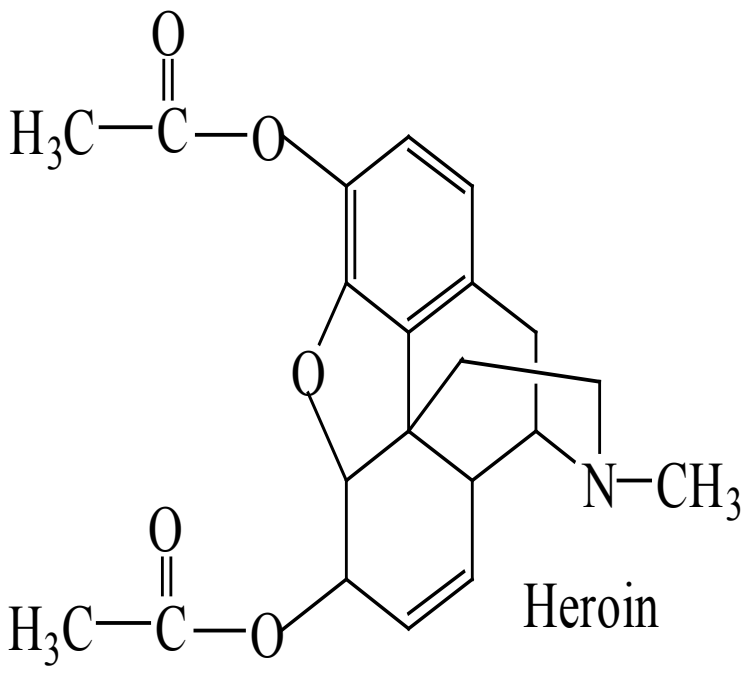

Natural impurities from opium 
Citation: Badria FA, El-Neketi M, Saad HA (2018) Forensic Analysis of a Confiscated Illicit Heroin Sample. Curr Res Bioorg Org Chem: CRBOC-105. DOI: 10.29011/ CRBOC -105. 100005
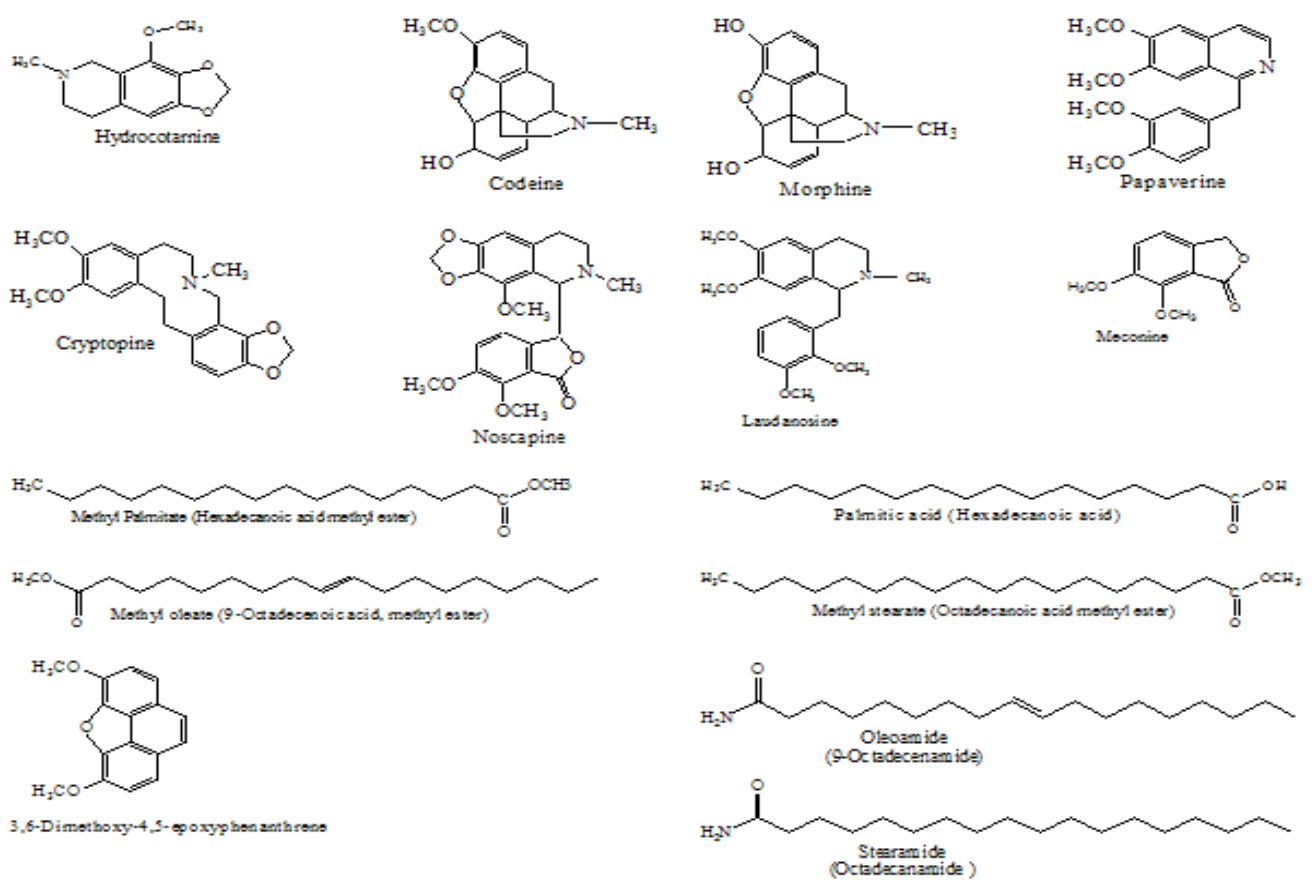

a group of n-alkanes:

$\begin{array}{ll}\text { n-Tricosane } & \text { Tetracosane } \\ \mathrm{C}_{23} \mathrm{H}_{48} & \mathrm{C}_{24} \mathbf{H}_{50} \\ \text { Octacosane } & \text { Nonacosane } \\ \mathrm{C}_{28} \mathrm{H}_{58} & \mathrm{C}_{29} \mathrm{H}_{60}\end{array}$

$\begin{array}{ll}\text { Pentacosane } & \text { Hexacosane } \mathrm{C}_{26} \mathrm{H}_{54} \\ \mathrm{C}_{25} \mathrm{H}_{52} & \\ \text { Triacontane } & \text { Hentriacontane } \\ \mathrm{C}_{30} \mathrm{H}_{62} & \mathrm{C}_{31} \mathrm{H}_{64}\end{array}$

Figure 8: Identified components of the illicit heroin sample.

\section{Impurities from manufacture process}

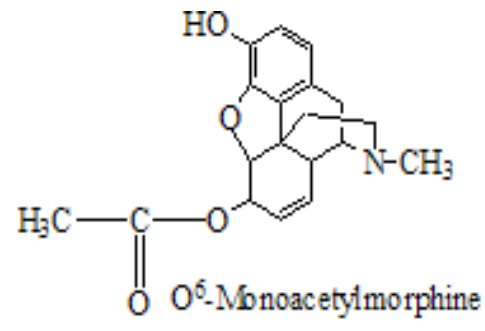

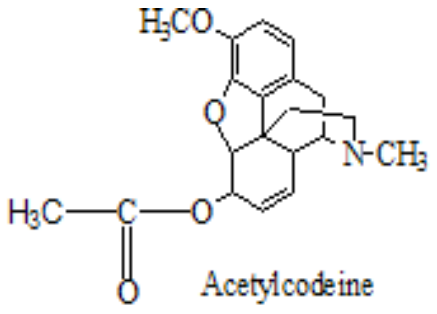<smiles>COc1cc2c(cc1OC)C(Cc1cccc(O)c1O)N(C(C)=O)CC2</smiles>

$\mathrm{N}$ - Acetylnorlaudanosine<smiles></smiles>

N- Acetylnornarcotine

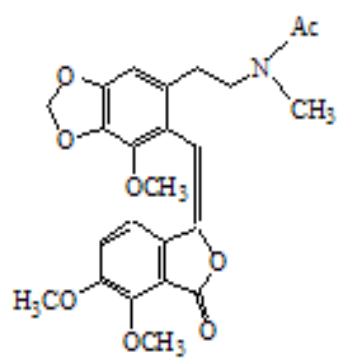

N-A cetylanhydronomarceine 


\section{Adulterants}
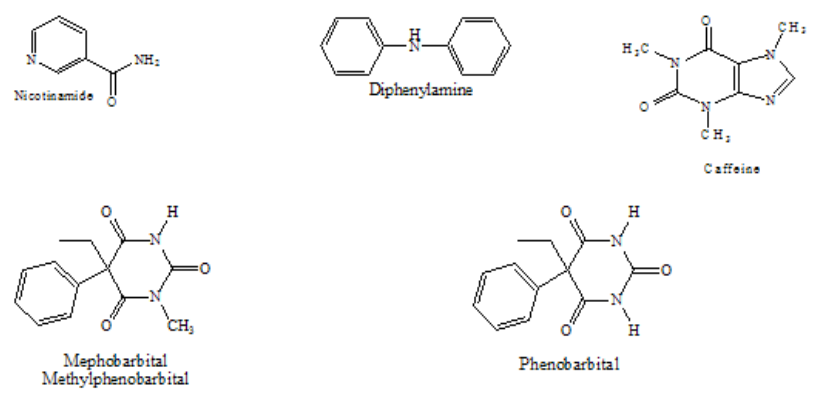

Figure 8: Cont.

\section{Discussion}

From the physical characters of illicit heroin, one may conclude the following:

1. It is crudely processed heroin sample as indicated by its dark brown or black color [43].

2. Its strong vinegar-like odor suggests the use of excess acetic anhydride as an acetylating agent or hydrolysis of heroin due to bad storage in humid atmosphere $[2,3,7]$.

3. Microscopic examination: of the sample revealed the presence of abundant starch granules suggested that the heroin sample under investigation was adulterated or diluted with starch [12]. Color, Solubility and Precipitation (Anion) tests: From the data cited in (Table 3), one could conclude the following:

- Presumptive color tests indicated the presence of opium alkaloids, heroin and caffeine which is commonly used as heroin adulterant.

- Solubility and precipitation tests showed that the heroin sample is present in the sulfate salt form diluted with starch which is insoluble in water and ethanol [1].

- The TLC data cited in (Table 4), revealed the presence of diacetylmorphine (heroin), O6-monoacetylmorphine (O6-MAM), Acetylcodeine (AC), morphine, codeine, noscapine, papaverine and caffeine in the heroin sample under investigation. The presence of caffeine in the heroin sample could indicate that it is of the South West Asian type characterized by its cutting with caffeine $[3,7]$.

\section{GC/MS}

GC/MS investigation of total heroin sample as well as neutral fraction revealed the presence of natural impurities from opium (24 components), impurities from the manufacturing process ( 5 components) and added adulterants ( 5 components). The identified components and their mean relative percentage are shown in (Table
7) and the structures of the identified components are illustrated in (Figure 8). From the fore mentioned data, one may conclude the following:

1.Heroin is present in a relatively low concentration $(0.6 \%)$ and O6-monoacetyl-morphine $\left(\mathrm{O}_{6}-\mathrm{MAM}\right)$ is the dominant component (34.4\%) which could be attributed to the following:

- The use of excess $\mathrm{H}_{2} \mathrm{SO}_{4}$ during the manufacture process of heroin that result in the hydrolysis of heroin to yield high percentage of $\mathrm{O}_{6}-\mathrm{MAM}$ and morphine $(>5 \%)$. These features are characteristics for poorly manufactured heroin which is the case of our sample [2].

- Partial hydrolysis of heroin to $\mathrm{O}_{6}$-MAM and then to morphine upon storage under humid condition [2,24,27] and the liberation of acetic acid 2 which is responsible for the strong vinegar-like odor of our sample.

- Partial hydrolysis of heroin to $\mathrm{O}_{6}$-MAM during injection into the GC system and/or during the silylation process [27].

2. $\mathrm{O}_{3}$-monoacetylmorphine $\left(\mathrm{O}_{3}\right.$-MAM) was not detected in our sample, as it is the product resulting from incomplete acetylation of morphine $[2,24]$. The absence of $\mathrm{O}_{3}$-MAM and the presence of high percentage $\mathrm{O}_{6}-\mathrm{MAM}(>10 \%$ relative to heroin) and morphine $(>1 \%$ relative to heroin) confirms that post-processing hydrolysis have occurred to our sample [27].

3. Thebaine was never detected in our heroin sample as it is unstable towards acetylation conditions. However, 3,6 Dimethoxy4,5 epoxyphenanthrene was detected as its decomposition product resulting from the reaction of thebaine with acetic anhydride $[3,27,38]$.

4. Noscapine concentration in the sample was found to be about $13.7 \%(<46 \%)$, which means that the present noscapine is a natural impurity from opium and is a non-intentionally added adulterant [21]. N-Acetylanhydronornarceine and N- Acetylnornarcotine were detected as the decomposition products of noscapine resulting from its reaction with acetic anhydride only or with acetic anhydride and oxygen, respectively [27].

5. The absence of 1-chloroheroin and 3-[1-(1carboxymethoxyethyl)]-6-acetylmorphine (the 2 route specific markers for acetyl chloride and ethylene diacetate) as components in the confiscated heroin sample excludes the use of acetyl chloride and ethylene diacetate as acetylating agents and support the use of acetic anhydride as an acetylating agent in the manufacturing process (3-United Nations, 1998; 5-Odell et al., 2006).

6.The lipid fraction consisting of fatty acid and fatty acid methyl esters was well resolved only on DB-1 column due to the low polarity of DB-1 in comparison to HP-5. The presence of this lipid fraction is characteristic for low quality crude morphine produced in South West Asia [33]. 
Citation: Badria FA, El-Neketi M, Saad HA (2018) Forensic Analysis of a Confiscated Illicit Heroin Sample. Curr Res Bioorg Org Chem: CRBOC-105. DOI: 10.29011/ CRBOC -105. 100005

7. It is important to note that, the fatty acid should be present in the original sample as volatile derivative with undetectable molecular ion peaks.

8. Polar compounds like morphine, monoacetylmorphine and Acetylcodeine were well resolved on polar stationary Phases HP-5 [44].

9. Caffeine and barbiturates (Phenobarbital and methyl phenobarbital) are characteristic as adulterants for South West Asian heroin. Caffeine was added to the heroin sample to enhance the amount of vaporized heroin without decomposition and improve the taste [45], while barbiturates were added as hypnotics [46].

Therefore, it could be concluded that the illicit heroin sample under investigation is a poorly manufactured sample, badly stored, adulterated and it is of the south West Asian type.

\section{Conclusion}

Application of physical and microscopical examination, color tests, Thin Layer Chromatography (TLC) and Gas Chromatography/ Mass Spectrometry (GC/MS) proved to be a very valuable tool for the characterization of the heroin sample under investigation, which has the following characteristics:

a. Physical characters: dark brown to nearly black, small granular pieces (chunks) having strong vinegar-like odor.

b. The absence of $\mathrm{O}_{3}$-MAM and the presence of high percentage $\mathrm{O}_{6}$-MAM content ( $>10 \%$ relative to heroin) and morphine $(>1 \%$ relative to heroin) confirms that post-processing hydrolysis have occurred to our sample.

c. The presence of heroin, morphine and $\mathrm{O}_{6}$-MAM at a concentration of about 37.0\%; Noscapine ( 13.7\%), papaverine $(\sim 2.2 \%)$ and Acetylcodeine $(\sim 7.9 \%)$ is characteristic for SWA type.

d. The relatively high concentration of noscapine $(13.7 \%)$ relative to morphine $(5 \%)$ confirms that the heroin sample is prepared via method 3 in which noscapine and morphine are in the ration 2:1 characteristic for crude morphine of SWA.

e. The presence of natural impurities such as meconine, noscapine, papaverine, cryptopine, laudanosine, fatty acids and fatty acid methyl esters confirms that it is a crudely processed heroin.

f. The presence of heroin as the sulfate salt.

g. The presence of abundant starch granules as adulterant.

h. The presence of caffeine and barbiturates (Phenobarbital and methylpheno-barbital) as adulterants is characteristic for SWA type.

Therefore, we can conclude that the heroin sample under investigation is: a poorly manufactured sample, prepared by acetylation with acetic anhydride, badly stored, adulterated, of the
South West Asian type and suggested to be abused by inhalation through smoking.

\section{References}

1. Sneader W (1998) Discovery of heroin. Lancet 352: 1697-1699.

2. Saad HA (2002) Narcotic Drugs and Psychotropic substances under international control. Arab Oublication House Egypt 1-66.

3. United Nations Publications (1998) Recommended methods for testing opium, morphine and heroin, Manual for Use by National Drug Testing Laboratories, ST/NAR/29/Rev.1.

4. United Nations Publications, World Drug Report, 2005, Volume I and

5. 5-Odell LR, Skopec J, McCluskey A (2006) A 'cold synthesis' of heroin and implications in heroin signature analysis Utility of trifluoroacetic/ acetic anhydride in the acetylation of morphine. Forensic Sci Int 164: 221-229.

6. 6-United Nations Office on Drugs and Crime (1953) The Analysis of Heroin Bulletin on Narcotics 2-007: 27-35.

7. United Nations Publication, "Recommended methods for testing Heroin, Manual for Use by National Narcotics Laboratories, ST/NAR/16 (1986).

8. McConnell Davis TW, Farmilo CG, Genest K (1962) Analysis of an impure heroin seizure. Bulletin on Narcotics 14: 47-57.

9. Lerner M, Mills A (1963) Analysis of impure heroin seizure. Bull Narc1007: $37-42$.

10. Clarke EGC (1986) Isolation and Identification of Drugs. 2nd Ed The Pharmaceutical Press, London.

11. Vignoli L, Guillot J, Gouezo F, Catalin J (1965) Study by thin-layer chromatography of several samples of crude morphine and of diacetylmorphine from illicit sources. Bull Trav Soc Pharm Lyon 9: 291-304.

12. May L, Kuo CT (1972) Total analysis of an illicit or "street" narcotic sample by thin-layer chromatography. BullNarc 24: 35-36.

13. Huizer H, Logtenberg H, Steenstra AJ (1977) Heroin in the Netherlands. Bull Narc 29: 65-74.

14. Mari F, Bertol E, Tosti M (1982) Heroin in the Florence area, Italy. Bull Narc 34: 37-44.

15. Navaratnam V, Fei HK (1984) Review of laboratory methods for the analysis of opiates and diluents in illicit drug traffic. Bulletin on Narcotics 36: 15-23.

16. Rajananda V, Nair NK, Navaratnam V (1985) An evaluation of TLC systems for opiate analysis. Bull Narc 37: 35-47.

17. Della Casa E, Martone GJ (1986) quantitative densitometric determination of heroin and cocaine samples by high-performance thin-layer chromatography. Forensic Sci Int 32: 117-120.

18. Ayyangar NR., Biswas S, Tambe A (1991) Separation of opium alkaloids by thin-layer chromatography combined with flame ionization detection using the peak pyrolysis method. J chromatogr 547: 538-543.

19. Pothier J, Galand N (2005) Automated multiple development thin-layer chromatography for separation of opiate alkaloids and derivatives. Journal of Chromatography A 1080: 186-191. 
Citation: Badria FA, El-Neketi M, Saad HA (2018) Forensic Analysis of a Confiscated Illicit Heroin Sample. Curr Res Bioorg Org Chem: CRBOC-105. DOI: 10.29011/ CRBOC -105. 100005

20. Besacier F, Chaudron_Thozet H, Rousseau Tsangaris M, Girard J, Lamotte A (1997) Comparative chemical analyses of drug samples: general approach and application to heroin. Forensic Sci Int 85: 113-125.

21. Klemenc S (2000) Noscapine as an adulterant in illicit heroin samples. Forensic Sci Int 108: 45-49.

22. Klemenc S (2001) In common batch searching of illicit heroin samplesevaluation of data by chemometrics methods. Forensic Sci Int 115: 43-52.

23. Myors RB, Crisp PT, Skopec SV, Wells RJ (2001) Investigation of heroin profiling using trace organic impurities. Analyst 126: 679-689.

24. El-Haj BM, Al-Amri AM, Ali HS (2004) Heroin profiling: mannitol hexaacetate as an unusual ingredient of some illicit drug seizures. Forensic Sci Int 145: 41-46.

25. Buryakov IA (2004) Express analysis of explosives, chemical warfare agents and drugs with multicapillary column gas chromatography and ion mobility increment spectrometry. Journal of Chromatography B 800: $75-82$

26. Sharma SP, Purkait, BC, Lahiri SC (2005) Qualitative and quantitative analysis of seized street drug samples and identification of source. Forensic Science International, 152: 235-240.

27. United Nations Publication, "Methods for Impurity profiling of Heroin and Cocaine", Manual for Use by National Drug Testing Laboratories, ST/NAR/35 (2005).

28. Oestreicher PM, Charles G, Farmilo, Levi L (1954) Part III B. Ultraviolet Spectral Data for Ninety Narcotics and Related Compounds. Bulletin on Narcotics7: 42-70.

29. Shamma M (1972) The isoquinoline Alkaloids, Chemistry and Pharmacology Academic Press, Inc, New York and London 25:378.

30. Levi L, Hubley CE, Hinbe RA (1955) Part IVB: Infrared Spectra of Narcotics and Related Alkaloids. Bull Narc 006: 42-45.

31. Balbaa SI, Hilal SH, Zaki AY (1981) Medicinal plant constituents. General Organization for University and School Books Cairo 280.

32. Strömberg L, Lundberg L, Neumann H, Bobon B, Huizer $\mathrm{H}$, et al. (2000) Heroin impurity profiling A harmonization study for retrospective comparisons. Forensic Sci Int 114: 67-88.

33. Neumann H (1984) Analysis of opium and crude morphine samples by capillary gas chromatography. Comparison of impurity profiles. $\mathrm{J}$ Chromatogr 315: 404-411.
34. NIST: NIST Chemistry webbook.

35. Hertz, HSK, Biemann (1976) On the occurrence of antipyrine in opium. Bulletin on Narcotics 1: 67-70.

36. GC liberary

37. Adams RP (1995) Identification of Essential Oil Components by Gas Chromatography/Mass Spectrometry. Allured Publishing Corporation, USA.

38. Allen AC, Cooper DA, Moore JM, Teer CB (1984) Thebaine Rearrangements: Non classical D Ring Migrations. J Org Chem 9: 3462-3465.

39. Theuns HG, Janssen RHAM, Seykens D, Salemink CA (1985) Alpinine, epialpinine and other alkaloids from Papaver Bracteatum. Phytochemistry $24: 581-584$.

40. Tatematsu A, Goto T (1965) Analysis of mixed drugs by mass spectrometry. I. Analysis of opium alkaloids. (1). Mass spectra of opium alkaloids and the allied compounds. Yakugaku Zasshi 85: 152-157.

41. Phillipson JD, Thomas OO, Gray Al, Sariyar G (1981) Alkaloids from Papaver armeniacum, P. fugax and P. tauricola. Planta Medica 41: 105-118.

42. Konda M, Shioiri T, Yamada SI (1975) Amino Acids and Peptides. XVII. A Biogenetic-type, Asymmetric Synthesis of (S)-Laudanosine from L-3 -(3,4-Dihydroxyphenyl) alanine by 1,3-Tansfer of Asymmetry. Chem Pharm Bull 23: 1025-1031.

43. Moore JM, Allen AC, Cooper DA (1986) Determination of Neutral Manufacturing Impurities in Heroin by Capillary Gas Chromatography with Electron Capture Detection after Reduction with Lithium Aluminum Hydride and Derivatization with Heptaflurobutyric Anhydride. Anal.Chem 58: 1003-1007.

44. Chiarotti M, Carnevale A, De Giovanni N (1983) Capillary gas chromatographic analysis of illicit diamorphine preparations. Forensic Sci Int 21: 245-251.

45. Kools JP from 0 to 600 centigrade in 2 seconds: Chasing the dragon. (From Mainline, special edition VIIIth International Conference on AIDS Amsterdam, July 1992)

46. Gomez J, Rodriguez A (1989) An evaluation of the results of a drug sample analysis. Bull Narc 41: 121-126. 